\title{
APORTACIONES SOBRE CAMBIOS EN LAS TEN- DENCIAS DE LAS VARIABLES CLIMÁTICAS EN LA MESETA CENTRAL ESPAÑOLA
}

\author{
M Teresa ORTEGA VILLAZÁN (maite@fyl.uva.es) \\ Carlos Gabriel MORALES RODRÍGUEZ (cmorales@fyl.uva.es) \\ Departamento de Geografía. Universidad de Valladolid \\ José Luis LABAJO SALAZAR (jll45@usal.es) \\ Departamento de Física General y de la Atmósfera. Universidad de Sala- \\ manca
}

Recibido: 25/01/2013

Devuelto: $11 / 06 / 2013$

Aceptado: 24/06/2013

RESUMEN: El interés creciente por los estudios acerca del Cambio Climático para delimitar y valorar su entidad en diferentes lugares del planeta, así como su evolución a lo largo de las últimas décadas y en un futuro, llevó desde 1997 al desarrollo de una línea de investigación sobre Cambio Climático, dentro del Grupo de Investigación Reconocido de Análisis del Clima de las Universidades de Salamanca (Departamento de Física General y de la Atmósfera) y de Valladolid (Departamento de Geografía), activo en la actualidad. Se presentan los resultados obtenidos de la investigación y producción científica llevada a cabo desde entonces, coincidiendo ahora con el cambio de su investigador principal, D. José Luis Labajo Salazar. El objetivo es mostrar las propuestas metodológicas seguidas a lo largo de estos años en el estudio de variables meteorológicas a partir de las cuales se pueda caracterizar el Cambio Climático en Castilla y León y en toda la meseta central española. Las investigaciones se han centrado en el análisis y modelado de las series temporales de temperaturas, precipitaciones y presión atmosférica a nivel de superficie, empleando técnicas estadísticas y de redes neuronales artificiales. Las aportaciones realizadas en el análisis de sus tendencias, frecuencias, comportamientos de valores extremos y olas de frío y calor, se sintetizan en el presente trabajo.

PALABRAS CLAVE: Cambio Climático, temperaturas, precipitaciones, presión superficial, homogeneización, anomalías, tendencias, valores extremos, Castilla y León, Plataforma central ibérica.

\section{CONTRIBUTIONS ON CHANGES IN VARIABLE WEATHER TRENDS IN SPANISH CENTRAL PLATEAU}

ABSTRACT: The growing interest in studies of Climate Change to identify and assess their entity in different parts of the world and its evolution over the last decades and in the future, led since 1997 to develop a line of research on Climate Change within the Climate Analysis Recognized Research Group in the Universities of Salamanca (Department of General Physics and Atmosphere) and Valladolid (Department of Geography), currently active. We 
present the results of scientific research and production carried out since, coinciding now with the change of principal investigator, D. José Luis Salazar Labajo. The aim is to show the methodological proposals followed over these years in the study of meteorological variables to characterize climate change in Castilla y León and throughout the Iberian central platform. The research has focused on the analysis and modeling of time series of temperature, precipitation and atmospheric pressure at the surface level, using statistical techniques and artificial neural networks. The contributions made in the analysis of trends, frequency of extreme values and behaviors of cold and heat waves, are summarized in this paper.

KEY WORDS: Climate Change, temperatures, precipitation, surface pressure, homogenization, anomalies, trends, extreme values, Castilla y Leon, Central Iberian Platform.

\section{INTRODUCCIÓN}

Durante los últimos dieciséis años uno de los objetivos del grupo de investigación de Análisis Climático de las universidades de Salamanca y Valladolid, ha sido tratar de establecer la evolución espacial y temporal del comportamiento extremo de determinadas variables meteorológicas en relación con el posible Cambio Climático actual. En los primeros trabajos los estudios se centraron en la región de Castilla y León $\left(94.227 \mathrm{~km}^{2}\right)$ y posteriormente se han hecho extensivos a todo el sector central ibérico, incluyendo las comunidades autónomas de Madrid y de Castilla-La Mancha hasta cubrir un total de algo más de $181.000 \mathrm{~km}^{2}$.

El grupo investigador no siempre ha sido el mismo, experimentando variaciones desde su consolidación en 1997. Los únicos que permanecen desde entonces son los tres firmantes de este artículo, pero también hay que destacar la labor desempeñada en su día por A. Piorno (hasta 2007) y posteriormente por A. L. Labajo (Departamento de Lenguajes y sistemas informáticos), Q. Martín (Departamento de Estadística) y más recientemente M. Egido (Departamento de Física General y de la Atmósfera).

El interés por el análisis y el comportamiento de las tendencias en distintas variables meteorológicas, objetivo fundamental de los estudios climáticos, se ha visto reforzado en las últimas décadas en su relación con el Cambio Climático. Así lo ponen de manifiesto numerosas publicaciones desde hace años (DEgAetANO, 1996; LABAJO y PIORNO, 1998; JONES et al., 1996; LABAJO y PIORNO, 1999...). En este sentido también se ha hecho extensivo este interés a la valoración de las dinámicas de los valores extremos, como han expresado más recientemente: "Muchos de los impactos críticos del clima están controlados por eventos extremos más que por valores medios" (SALINGER et al., 2001:1437)

El hombre se adapta bien a las condiciones climáticas medias, a las pequeñas variaciones dentro de su amplio elenco de posibles manifestaciones cli- 
máticas (los tipos de tiempo habituales o predominantes). Sin embargo, nuestra capacidad de respuesta disminuye progresivamente a medida que las condiciones se hacen más extremas (los tipos de tiempo menos frecuentes o irregulares, que no anómalos). Cambios en el comportamiento de los valores medios y de los extremos pueden hacer que sus impactos climáticos sean más habituales que infrecuentes. Esto refuerza la necesidad de su estudio y que éste se haga en intervalos de observación largos, pues son todos los posibles tipos de tiempo (habituales y extremos) los que crean ese ambiente climático permanente que define lo que es el clima en sí.

Así pues, el análisis del comportamiento espacio-temporal de la ocurrencia de fenómenos atmosféricos de carácter extremo constituye uno de los elementos clave en la caracterización del Cambio Climático actual a partir de datos instrumentales. De ahí que desde hace años diversos organismos internacionales (el IPCC entre otros), hayan llamado la atención a la comunidad científica para que centren su atención en estas líneas de investigación.

Los análisis llevados a cabo en las últimas décadas sobre el comportamiento espacio-temporal de distintas variables meteorológicas no siempre han dado conclusiones fiables ni contundentes. Para el caso de las temperaturas, sean máximas, mínimas o medias, los resultados son bastante buenos (AESAWY et al., 1998; MAHERAs et al., 1999...), pero no sucede lo mismo con las precipitaciones. La gran variabilidad con la que se muestra este meteoro a cualquier escala (espacial y temporal) dificulta bastante el análisis de su comportamiento. A nivel global existen modelos válidos para sus distintos estados de agregación, pero todo se complica al analizar su fase líquida y sólida a escalas menores a fin de establecer regionalizaciones posibles de esta variable (LABAJO y PIORNO, 2001). Su alta variabilidad en la distribución espacial favorece la realización de estudios sobre espacios con características geográficas uniformes.

Igualmente, desde hace décadas se orienta la investigación al análisis del comportamiento extremo de las precipitaciones (LIEBMANN et al., 2000; BRUNETTI et. al, 2000). Si admitimos a nivel global un incremento entre un 2-4\% de las precipitaciones intensas ante la mayor actividad tormentosa y alta inestabilidad a gran escala (Tercer Informe IPCC, 2001), es obvio el reparar en la frecuencia de aparición de este tipo de fenómenos extremos.

También preocupa el relacionar los posibles cambios térmicos y pluviométricos con los de la presión atmosférica a nivel regional pues las variaciones que ésta experimenta a lo largo del tiempo influyen directamente en el comportamiento de otras variables meteorológicas. La alteración o no de la fre- 
cuencia de distintos campos de presión marca el ritmo del tiempo atmosférico, y por ende, del comportamiento de temperaturas y precipitaciones.

Las investigaciones realizadas hasta ahora muestran que el Cambio Climático actual se justifica más por la variación en el comportamiento de valores extremos que por la de valores normales climáticos. Es más, son los que ponen de manifiesto que el clima ha de analizarse en intervalos temporales más dilatados y que, a largo plazo, son los responsables de la evolución temporal que experimentan los valores medios globales.

Dichos fenómenos están contribuyendo a la ocurrencia de graves desastres por el aumento de la peligrosidad climática, lo que favorece mayores situaciones de riesgo para el hombre. Es evidente el enorme influjo que las condiciones climáticas extremas tienen sobre el normal desarrollo de las diversas actividades socio-económicas así como del propio ser humano, con efectos catastróficos en ocasiones (olas de frío, olas de calor, sequias...). Por ello, el tratar de conocer posibles cambios en la dinámica de estos procesos extremos ha guiado las líneas de investigación desarrolladas, desde hace más de un quindenio, por este grupo de investigación ante el gran influjo que ejerce en la planificación de actividades y ordenación del territorio.

\section{OBJETIVOS}

Los objetivos se han ido ampliando con el paso del tiempo en virtud de los resultados que se han ido obteniendo, tanto en lo referente a la tipología de las variables analizadas y sus manifestaciones como del ámbito de estudio. Una secuencia lógica en la que se pueden destacar las siguientes etapas:

Primero el establecimiento, a partir de datos instrumentales, de una base de datos diarios de temperaturas, precipitaciones y presión homogeneizadas y de calidad contrastada, de todos los observatorios elegidos con los que poder trabajar de forma coherente y fiable. Con ellas se han construido las correspondientes series estacionales y regionales. Lógicamente dichas bases de datos se han ido actualizando en su número de años de observación con el paso del tiempo.

Segundo, y a partir de ellas, se han analizado la posible existencia de tendencias en las diversas variables meteorológicas estudiadas.

Tercero, tras identificar y localizar los eventos extremos, se ha establecido el comportamiento espacial y temporal de la frecuencia con que éstos se producen, modelando su distribución a lo largo del año, su intensidad, duración y frecuencia (olas de calor y frío) en la región de Castilla y León primero, y más tarde en el sector central ibérico. 
Estos objetivos se han ido desarrollando a través de los diferentes proyectos de investigación realizados por el grupo durante este tiempo (CUADRO 1).

Cuadro 1. Evolución de la Investigación del Grupo de Cambio Climático (USalUVa)

\begin{tabular}{|c|c|c|c|}
\hline Fecha & Objetivos/Resultados & $\begin{array}{c}\text { Ámbito estu- } \\
\text { dio }\end{array}$ & $\begin{array}{l}N^{o} \text { Publi- } \\
\text { caciones }\end{array}$ \\
\hline 1998-2003 & $\begin{array}{l}\checkmark \text { Análisis del comportamiento temporal de } \\
\text { la presión media anual y de la temperatura } \\
\text { mínima media anual. } \\
\checkmark \text { Control de calidad de datos de precipita- } \\
\text { ciones y temperaturas (máximas y míni- } \\
\text { mas) y construcción de bases de datos ho- } \\
\text { mogéneas. } \\
\checkmark \text { Análisis de tendencias de variables térmi- } \\
\text { cas. }\end{array}$ & $\begin{array}{l}\text { Castilla y } \\
\text { León }\end{array}$ & 8 \\
\hline 2004-2006 & $\begin{array}{ll}\checkmark & \text { Comportamiento de valores extremos de } \\
\text { presión atmosférica. } \\
\checkmark \\
\text { Tendencias recientes de las frecuencias de } \\
\text { valores extremos de las temperaturas y de } \\
\text { la presión. }\end{array}$ & $\begin{array}{l}\text { Castilla y } \\
\text { León } \\
\text { Madrid } \\
\text { Castilla-La } \\
\text { Mancha } \\
\end{array}$ & 5 \\
\hline 2007-2012 & $\begin{array}{l}\checkmark \quad \text { Aplicación de modelos de redes neurona- } \\
\text { les a las temperaturas medias mensuales } \\
\text { máximas y mínimas. } \\
\checkmark \quad \text { Evolución de los días extremadamente } \\
\text { fríos y cálidos. } \\
\checkmark \quad \text { Tendencias de la frecuencia de olas de frío } \\
\text { y olas de calor. }\end{array}$ & $\begin{array}{l}\text { Castilla y } \\
\text { León } \\
\text { Madrid } \\
\text { Castilla-La } \\
\text { Mancha }\end{array}$ & 8 \\
\hline
\end{tabular}

En definitiva, los objetivos perseguidos han tratado de constatar si ha existido o no un cambio apreciable en las anomalías de las temperaturas, precipitaciones y presión atmosférica superficial; en la producción de la frecuencia de eventos extremos en las distintas variables analizadas; evaluar la variabilidad interanual e interestacional en el comportamiento de las mismas, así como delimitar el período del año en el que esto es posible y qué territorios del interior peninsular son más afectados.

Por otro lado, el trabajar primero la información climática de Castilla y León y posteriormente ampliarlo a todo el sector central de España se ha hecho con el fin de establecer comparaciones en el comportamiento de las distintas variables, y poder tener una visión más completa de la evolución climática del interior del país. Ante todo se contribuye a completar los patrones de comportamiento climático y a cuantificar el Cambio Climático actual en una extensa superficie del interior peninsular (casi el 40\%). 


\section{METODOLOGÍA DE ESTUDIO}

Desde el principio se procede a la selección de aquellos observatorios de la red que la Agencia Estatal de Meteorología (AEMET) tiene establecida en las diversas CC.AA analizadas, cuyas series climáticas son más fiables, largas, en formato digital, y que ofrecen mayores garantías de calidad. Según el tipo de investigación el número de observatorios elegido varía, aunque siempre se busca una cobertura espacial uniforme y representativa (FIG.1). El intervalo de tiempo estudiado siempre es el máximo que permita la longitud de las series seleccionadas.

El control de calidad de las series sigue varios criterios, tales como longitud o tamaño de la muestra, series más completas de datos diarios, coincidencia temporal de las observaciones entre estaciones (al menos 20 años con un período común), a fin de poder establecer comparaciones. Se descartan aquellas series con demasiadas lagunas (más del 15\% de la serie).

Figura 1. Mapa hipsométrico y de localización de observatorios de Castilla y León

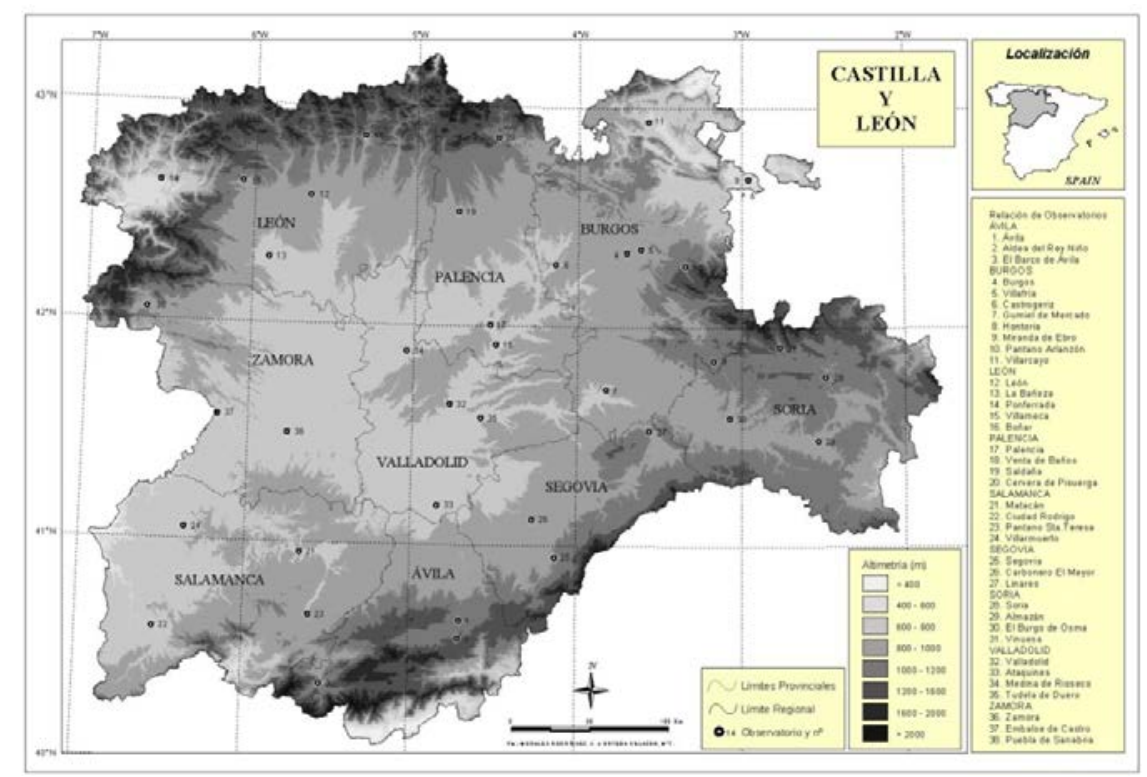

FUENTE.: MORALES et al. (2005)

En una primera fase se depuran las series aplicando a cada una de ellas filtros lógicos para detectar valores erróneos (outliers, datos que se apartan $\left.4 \pm \sigma^{2} / \bar{M}\right)$. A continuación se realiza el relleno de los huecos que existen ini- 
cialmente o de los nuevos tras el proceso de depuración. Primero se realiza a partir de las fuentes originales de obtención de los datos u otras posibles fuentes (metadata) con información complementaria, y después, se rellenan con técnicas estadísticas de regresión múltiple, empleando siempre un mínimo de cuatro observatorios y las series mejor correlacionadas.

En la fase siguiente se homogeneízan las series de datos a través de los tests estadísticos más utilizados y contrastados (como Standard Normal Homogeneity Test for Shift Detection de ALEXANDERSSON et al., 1997), y de los establecidos específicamente en cada trabajo, lo que ha llevado en ocasiones a elaborar un software concreto para automatizar sus diversas aplicaciones Siempre se han utilizado las estaciones mejor correlacionadas con la candidata (coeficientes de correlación $>0.8$ ), seleccionando las seis mejores. Los datos obtenidos permiten detectar inhomogeneidades, tanto de salto como de tendencia, y el proceso de homogeneización se aplica en cada observatorio a todas las variables (temperatura, precipitación...) en sus valores medios mensuales, estacionales y anuales (FIG. 2). Lógicamente las nuevas series construidas son sometidas al correspondiente análisis de residuos para su validación.

Una vez superados todos estos procedimientos se obtienen las series de datos finales homogeneizados de las distintas variables (LABAJO et al. 2000; MORALES et al. 2000). A partir de ellas se realiza el estudio sobre la evolución climática de la región y se determinan qué eventos se pueden calificar de "extremos". Con tal fin se concretan qué umbrales marcan esos valores de temperaturas máximas diarias, mínimas diarias, precipitación en 24 h., presión alta, olas de frío, olas de calor, etc..., y la persistencia de cada situación. En función de ello se determinan las frecuencias anuales y estacionales de eventos extremos y su duración para cada una de las series de datos y para cada región analizada.

Conviene apuntar que la variable temperatura ha sido más fácil de trabajar que las precipitaciones y la presión, al disponer de información más completa, extensa y fidedigna así como un comportamiento espacio-temporal menos aleatorio. Hecho que también ha favorecido la realización de forma paralela y más recientemente, de un análisis temporal del comportamiento de las temperaturas medias mensuales mediante la utilización de un modelo de Red Neuronal Artificial (LABAJO, 2007; LABAJO et al., 2011). Éste ha demostrado su capacidad para modelar de forma no lineal los valores futuros, incluidos los extremos, de esta serie temporal a partir únicamente de valores pasados de esa misma serie. El modelo generado a partir de RNA es de gran fiabilidad pudiéndose utilizar como un "método de calidad suficiente para el re- 
lleno de lagunas de las series temporales de datos climáticos” (LABAJO, et al. 2007).

Figura 2. Ejemplo del proceso de homogeneización aplicado a las temperaturas máximas de verano en la estación de Zamora
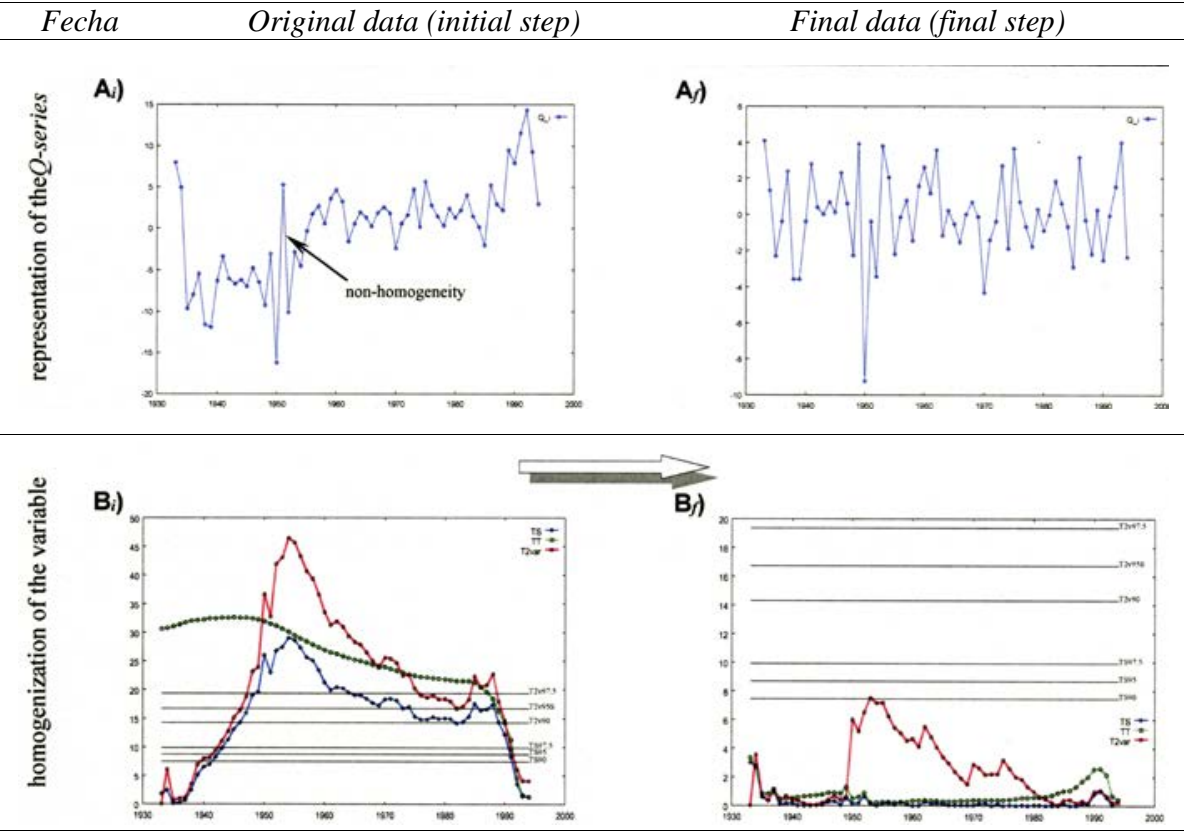

FUENTE: Elaboración propia

A partir de los datos diarios se construyen las series de anomalías diarias de las variables analizadas en sus distintos períodos de observación. Para ello, tras ordenar las series de datos de cada observatorio por días (todos los días del 1 , todos los días $2 . .$. ), mes (todos los meses de enero...) y año (todos los años del período analizado), se calculan las diferencias entre cada valor diario de la serie y el valor promedio calculado para cada día del año en $\operatorname{los} n$ años de observación.

El trabajo con series de anomalías permite cotejar directamente los datos de unos observatorios con otros, se elimina la necesidad de reducir los datos a un nivel de referencia determinado y, además, se filtra la periodicidad anual que afecta a los valores (estacionalidad).

A partir de las series de anomalías se construyen las series regionales. Aunque se han empleado diversos métodos de interpolación de valores espaciales (kriging, regresión polinómica, método Shepard, triangulación con interpolación linear), normalmente ha sido el del "inverso de la distancia pon- 
derada" el que mejor se adecua a la malla construida (entre los $40^{\circ}-43^{\circ}$ latitud y los $2^{\circ}-7^{\circ}$ longitud $\mathrm{W}$, cuadrículas de $1^{\circ} \mathrm{x} 1^{\circ}$ de latitud y longitud para Castilla y León, por ejemplo) y al análisis de las distintas variables (BRUNET et al. 1999). Se trata de un método sencillo de interpolación sobre una red espacial de cuadrículas regulares que presenta unos resultados de alto grado de bondad como ponen de manifiesto trabajos como el de KARL et al. (1994).

El cálculo de las series promedio de anomalías para cada cuadrícula se obtiene a partir de los observatorios incluidos en cada una de ellas ponderándolos en función del inverso de su distancia al centro de ella, mediante la siguiente expresión:

$$
\Delta \mathrm{V}_{c}=\frac{\sum_{i=1}^{n} d_{i} \Delta \mathrm{V}_{i}}{\sum_{i=1}^{n} d_{i}}
$$

Donde $\Delta \mathrm{V}_{c}$ es la anomalía de la variable (temperatura...) interpolada en el centro de cada cuadrícula; $\Delta \mathrm{V}_{i}$ es la anomalía de la variable analizada, establecida en cada observatorio de estudio; $d_{i}$ es el inverso de la distancia al centro de su cuadrícula del observatorio $i$ y $n$ el número de estaciones comprendidas en la cuadrícula.

A partir de los datos promedio de cada cuadrícula, se obtienen las series de valores promedio para toda la región $\left(\mathrm{V}_{r}\right)$, repitiendo este proceso para las tres variables en intervalos temporales mensuales, estacionales y anuales. Igualmente, se realiza un análisis gráfico del comportamiento de las diversas series de anomalías para cada una de las variables (FIGURAS 3 y 4).

Así pues, mediante técnicas de agregación espacial se generan series regionales representativas, que después de ser contrastadas con las series individuales de los observatorios, permitan realizar su análisis temporal. Este se realiza a partir de esas series regionales de anomalías absolutas o estandarizadas (diarias, estacionales, anuales) respecto a un intervalo de referencia común (JONES y HULME, 1996; VINNIKOV et al., 1990). En el estudio del comportamiento temporal se utilizan diversos test estadísticos (Spearman, Mann-Kendall...), para confirmar la existencia de tendencias a un nivel de confianza superior al 95\%.

Posteriormente se determina la zonación de aparición de sucesos climáticos extremos en cada región y se analiza su comportamiento. Para ello es preciso construir las correspondientes series de datos de valores extremos a partir de las series regionales de anomalías, seleccionando aquellos que ex- 
Figura 3. Evolución temporal de las anomalías de precipitación total, estacional (invierno y primavera) y anual.
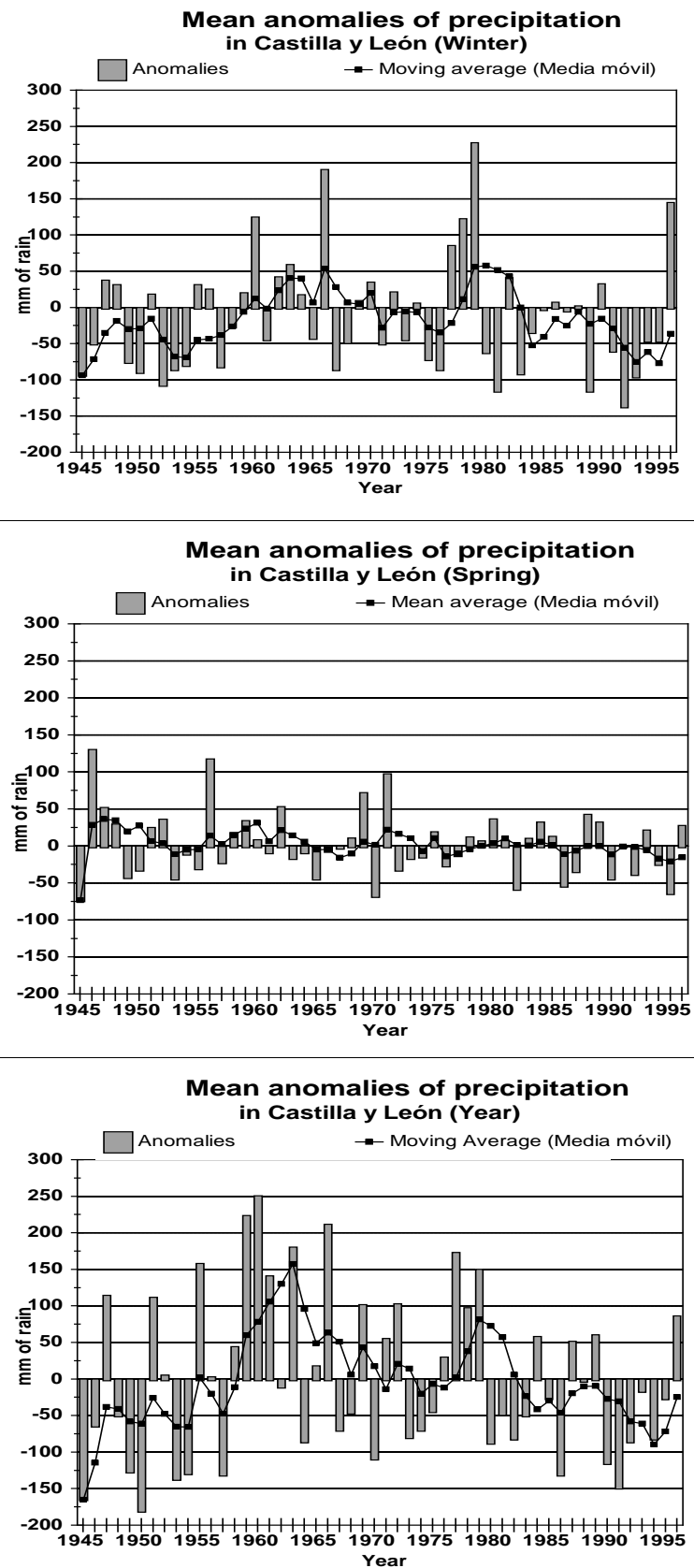

FUENTE.: LABAJO y PIORNO (2001)

Polígonos. Revista de Geografía, 24 (2013); 43-75 
Figura 4a. Evolución estacional y anual de las anomalías de temperatura regionales en Castilla y León, en el periodo 1945-1996. Winter.
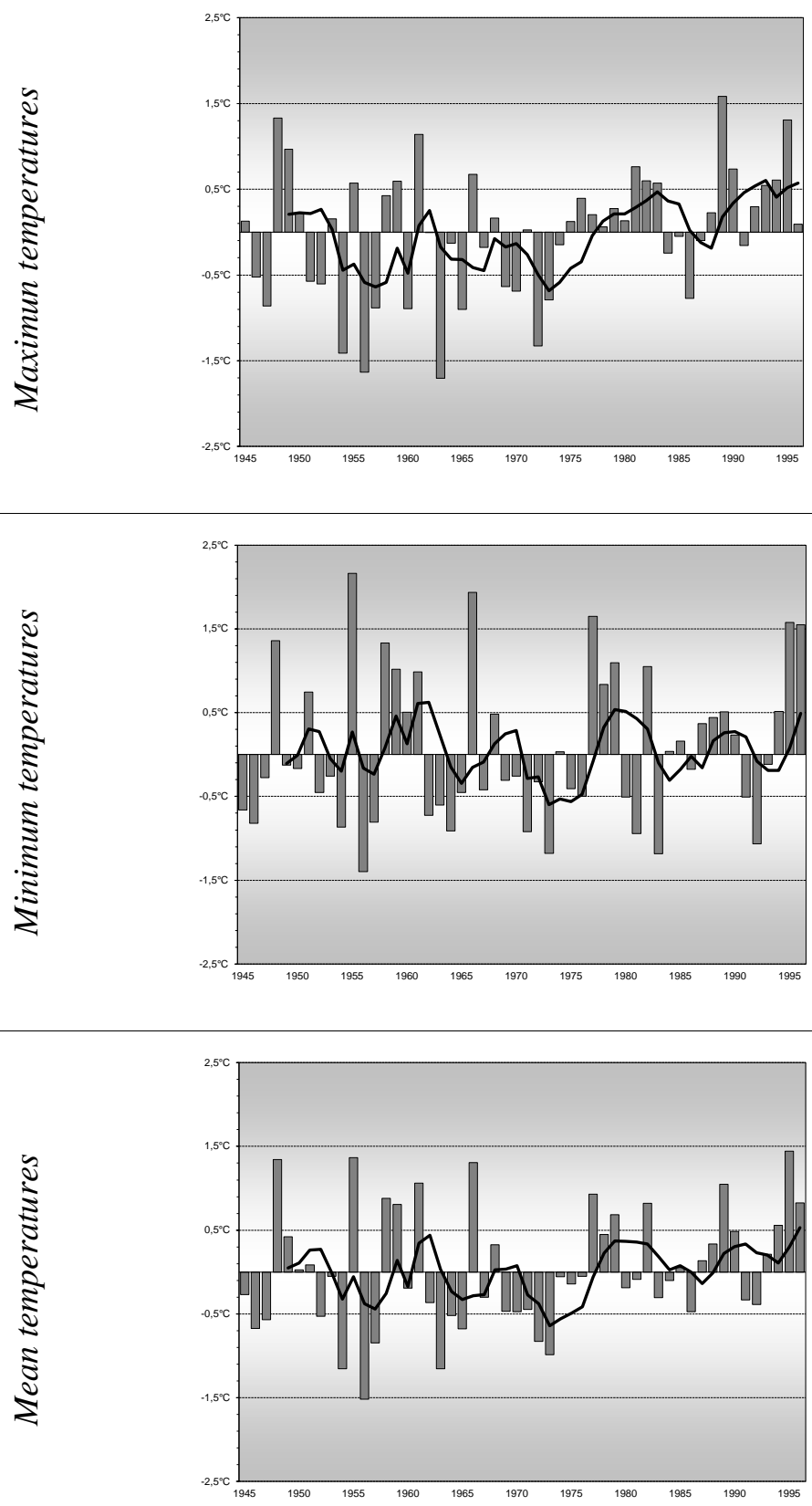

FUENTE.: MORALES et al. (2005)

Polígonos. Revista de Geografía, 24 (2013); 43-75 
Figura 4b. Evolución estacional y anual de las anomalías de temperatura regionales en Castilla y León, en el periodo 1945-1996. Spring.
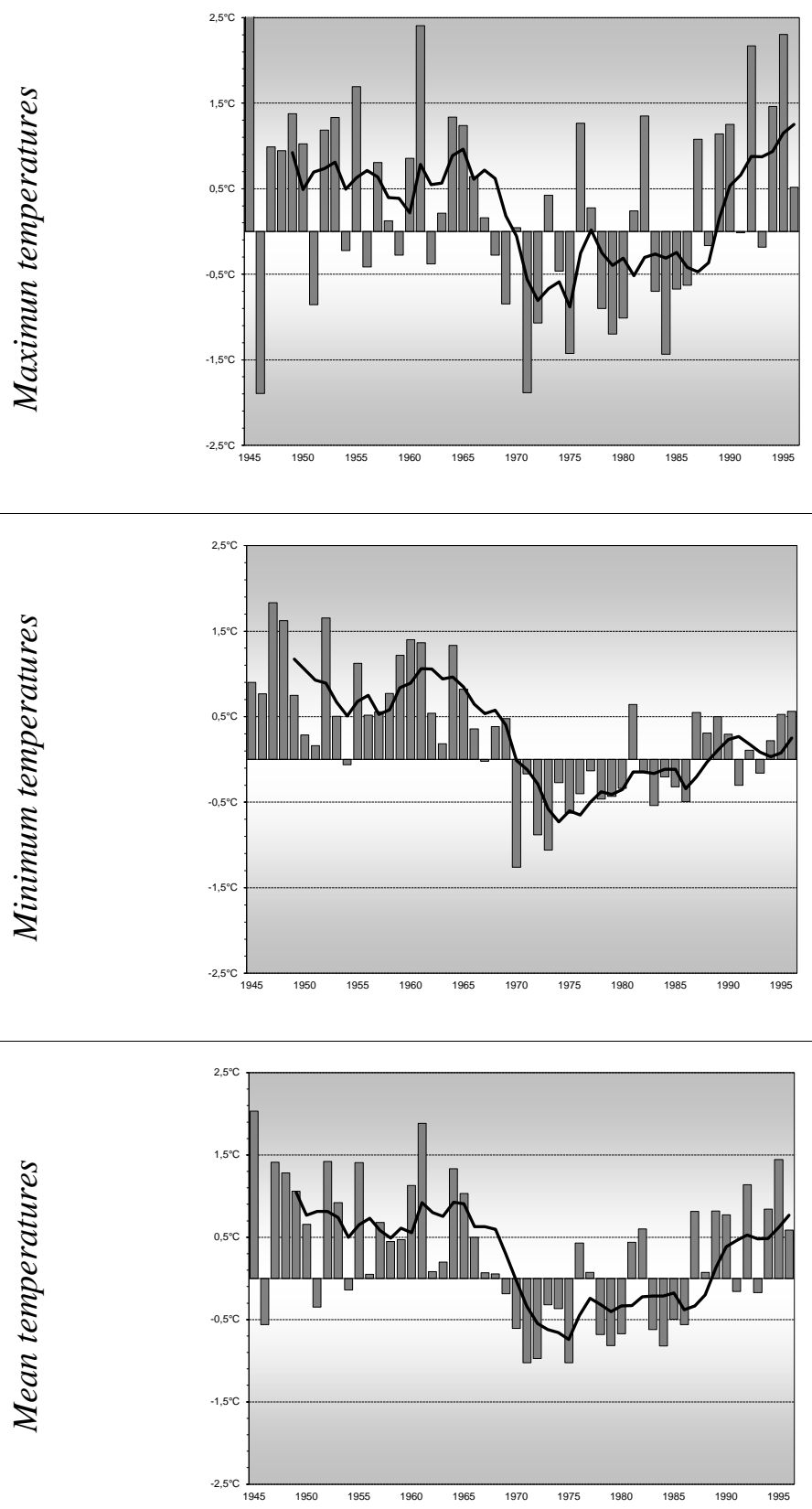

FUENTE.: MORALES et al. (2005)

Polígonos. Revista de Geografía, 24 (2013); 43-75 
Figura 4c. Evolución estacional y anual de las anomalías de temperatura regionales en Castilla y León, en el periodo 1945-1996. Autum.
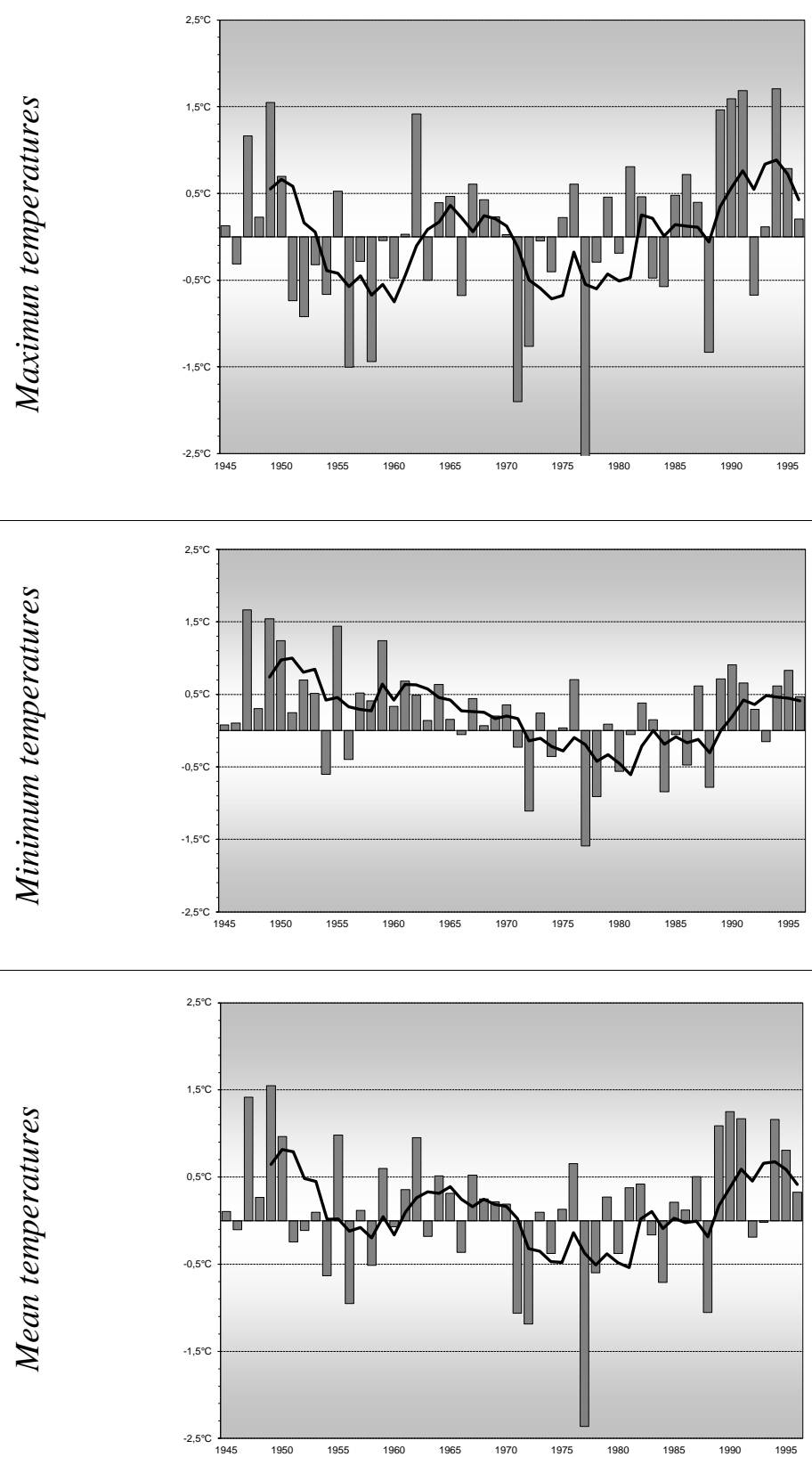

FUENTE.: MORALES et al. (2005) 
Figura 4d. Evolución estacional y anual de las anomalías de temperatura regionales en Castilla y León, en el periodo 1945-1996. Summer.
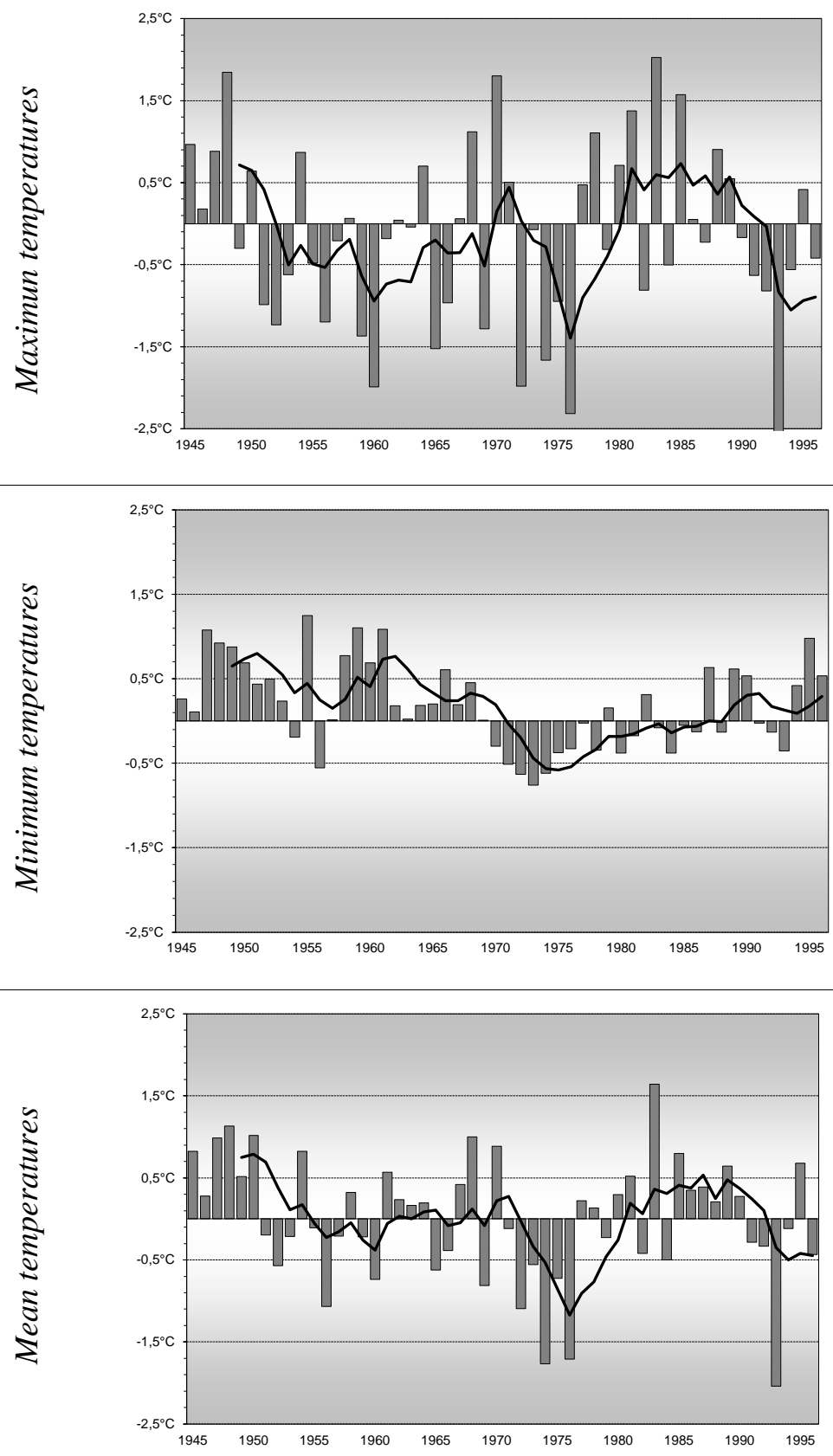

FUENTE.: MORALES et al. (2005)

Polígonos. Revista de Geografía, 24 (2013); 43-75 
Figura 4e. Evolución estacional y anual de las anomalías de temperatura regionales en Castilla y León, en el periodo 1945-1996. Year.
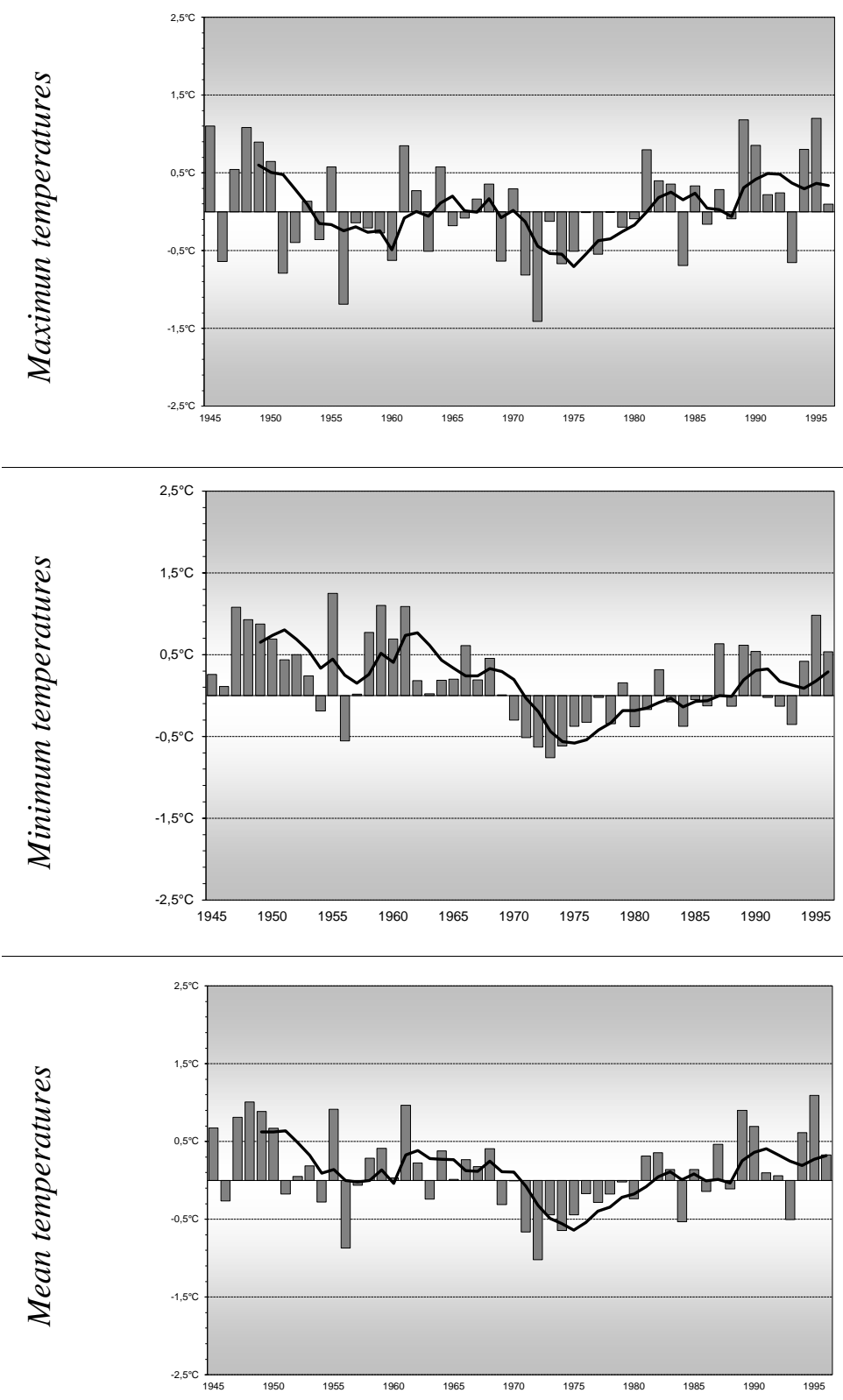

FUENTE.: MORALES et al. (2005) 
cedan unos umbrales que los definan, tanto para los valores más altos como para los más bajos, en cada una de las variables analizadas.

Estos umbrales de valores extremos se pueden establecer a partir de diferentes criterios. En esta ocasión, se han considerado los percentiles en su valores correspondientes a $\mathrm{P}_{05}$ (para los extremos más bajos) y $\mathrm{P}_{95}$ (para los extremos más altos), dado que está siendo empleado profusamente tanto para definir intervalos de clase en las serie de variables (LANA et al., 2003) como para determinar umbrales de valores extremos (DEGAETANO y ALLEN, 2002; GRIFFITHS, et al., 2003). En otros casos, se pueden usar criterios más específicos, como sumar o restar al valor medio de las anomalías de cada serie de valores la desviación típica correspondiente (empleado en la presión, LABAJO et al. 2004).

Definidos así los umbrales de valores extremos, se construyen las series de frecuencias anuales de días con valores de anomalías por debajo y por encima de ellos. El análisis de las variaciones temporales de las frecuencias de producción de los eventos extremos permite reconocer distintas tendencias, tanto totales (todo el período de estudio) como parciales (distintos períodos si se marcan cambios de tendencia). Las tendencias anuales y estacionales se han analizado, al igual que el caso anterior, a partir de técnicas estadísticas (tendencias lineales, filtros de paso bajo, establecimiento de puntos de cambio de tendencia...), siempre dentro de niveles de confianza altos (del 95\%).

Finalmente también se ha analizado el comportamiento de las olas de frío y de las olas de calor durante el período 1961-2010, para todo el sector central español (Castilla y León, Madrid y Castilla-La Mancha), a partir de las series de datos diarios de anomalías de temperatura máxima y mínima (ATMAD, ATMID). El trabajar con las series de anomalías ha permitido realizar un estudio anual sin tener que restringirlo a una estación determinada del año (olas de frío sólo en invierno y de calor sólo en verano) al eliminarse la estacionalidad y verse reducida la variabilidad térmica. El estudio del comportamiento temporal de esos episodios es necesario definirlo en función de las mismas variables climáticas en todo el año, al margen de los efectos sobre los ecosistemas o la actividad humana (LABAJO, A.L. et al., 2012a y 2012b). Son pues, definiciones estrictamente climatológicas según muestran los datos.

La definición de días extremadamente fríos (DEF) lo marcan aquellos cuyos valores de ATMAD y ATMID son inferiores, simultáneamente, a los valores umbrales que marcan los percentiles $05\left(\mathrm{P}_{05}\right)$ de ambas series (máximas y mínimas). No obstante, la definición de olas de frío se establece a partir de los umbrales que define el percentil $10\left(\mathrm{P}_{10}\right)$, al considerarse excesivamente 
restrictivo el $\mathrm{P}_{05}$. Este último ayuda a determinar cuáles han sido los episodios de frío más intensos (ALLEN R. et al., 2001). Así, se entiende como ola de frío una sucesión de dos o más días consecutivos en los que las ATMAD y las ATMID son inferiores simultáneamente a los umbrales establecidos. (FIGURA 5).

Figura 5. Evolución temporal de la frecuencia anual de olas de frío $\left(P_{10}\right)$ y de calor $\left(P_{90}\right)$ en el período 1961-2010

Evolución temporal del número de olas de frio entre 1961-2010 ATMAX Y ATMIN <P10

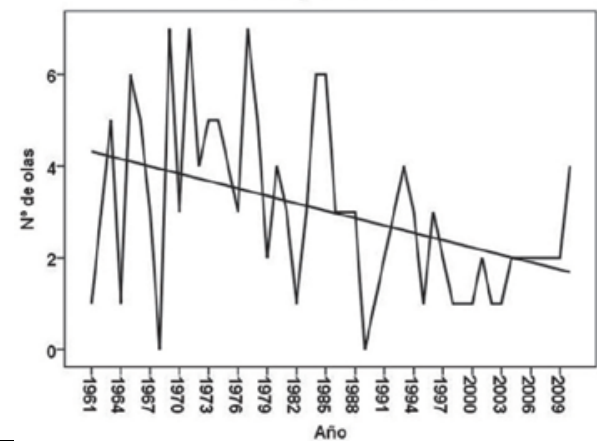

Evolución temporal del número de olas de Calor entre 1961-2010

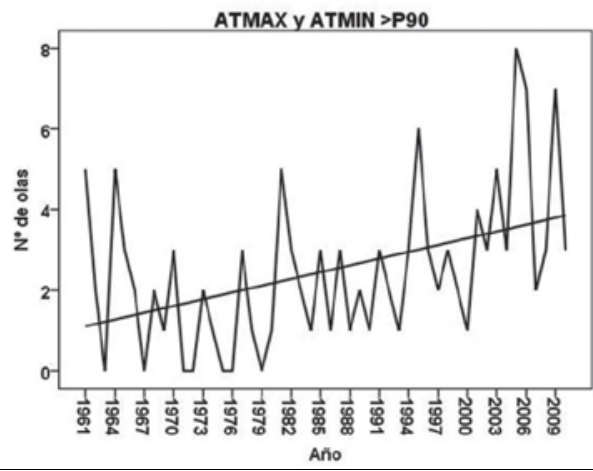

FUENTE: LABAJO, A.L. et al. 2012a (olas de frio) y 2012b (olas de calor)

Por su parte, se consideran días extremadamente cálidos (DEC) aquellos donde las ATMAD y ATMID superan, simultáneamente, los valores de los umbrales determinados por los percentiles $95\left(\mathrm{P}_{95}\right)$ de ambas series (máximas y mínimas). No obstante, la delimitación de olas de calor se establece, de forma análoga al caso anterior, a partir de los umbrales que define el percentil $90\left(\mathrm{P}_{90}\right)$, al considerarse excesivamente restrictivo el $\mathrm{P}_{95}$. Este último ayuda a determinar cuáles han sido los episodios de calor más intensos. Se considera la existencia de ola de calor cuando ambas variables (ATMAD y ATMID) superan los umbrales determinados por el percentil 90 durante dos días consecutivos como mínimo. (FIGURA 5).

Dos o más olas de frío o de calor separadas por un intervalo de un día se consideran que forman parte de la misma ola siempre y cuando una de las dos variables (ATMAD o ATMID) sea inferior o superior al valor umbral correspondiente en ese día intermedio. Una vez identificadas las olas de frío y de calor, se determinan sus frecuencias anuales y mensuales, y a partir de ellas el análisis de las tendencias de sus series.

La secuencia metodológica de la investigación llevada a cabo durante estos años queda pues resumida en la siguiente tabla (CUADRO 2). 


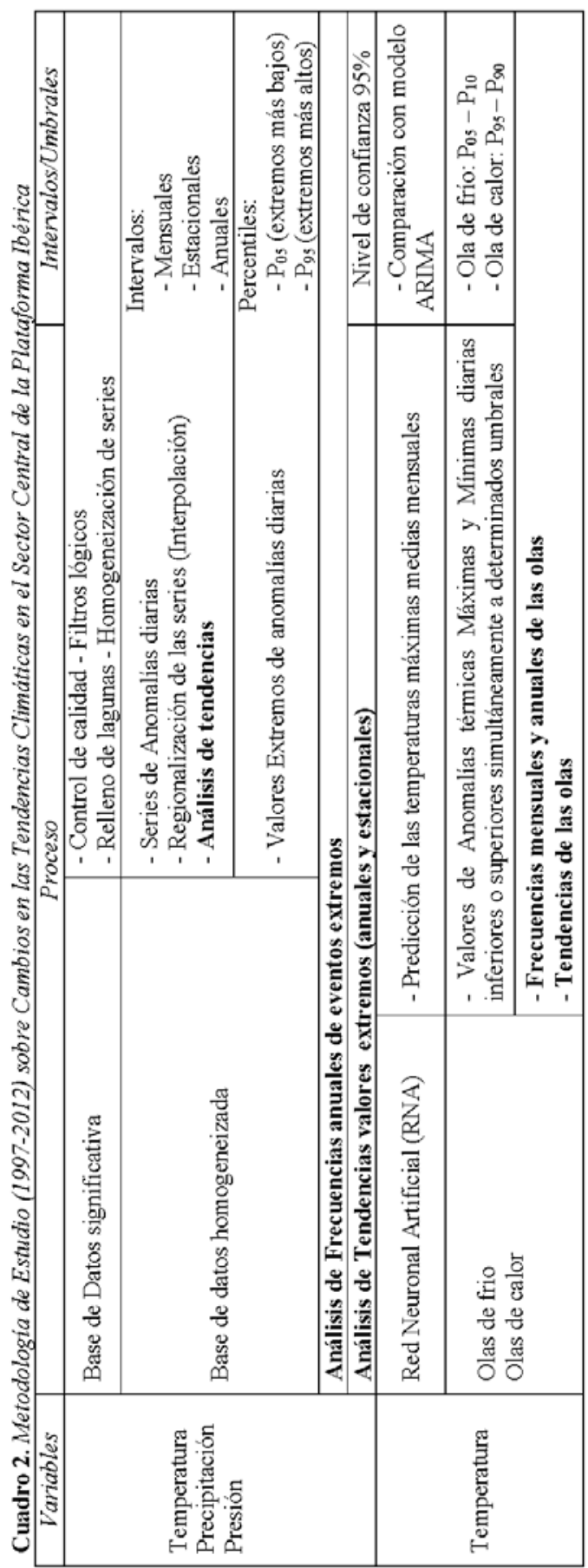

Polígonos. Revista de Geografía, 24 (2013); 43-75 


\section{RESULTADOS DE LAS INVESTIGACIONES}

\section{IV.1. En las temperaturas}

Las temperaturas constituyen una variable meteorológica de primer orden, representativa de los caracteres climáticos de un territorio. Es más, a través de ella se detectan mejor las posibles variaciones en el clima. El Cambio Climático actual, iniciado al comienzo de la era industrial como consecuencia del incremento paulatino del vertido a la atmósfera de gases efecto de invernadero (como causa más probable), está totalmente probada por un incremento medio, a escala global, de la temperatura media anual del aire en superficie. El tercer informe del IPCC (2001) ya cifra este aumento en torno a $0,6^{\circ} \mathrm{C}$ (con un intervalo de confianza de $\pm 0,2$ ) desde fines del siglo XIX. En este período se han detectado dos períodos de calentamiento (1910-1945) y (1976-2000), con un ritmo de aumento de las temperaturas de $0,15^{\circ} \mathrm{C} /$ decenio, y un período de enfriamiento (1946-1975), como muestra dicho informe. Estos períodos parecen estar admitidos por todos los investigadores, si bien los límites de los mismos pueden variar según territorios. Para el caso de Castilla y León, el año que marca el comienzo del último período cálido es 1972 (LABAJO et. al., 1998).

Los estudios sobre el comportamiento de las temperaturas en los últimos 65 años se han planteado a partir del análisis de las tendencias de las anomalías térmicas regionales apreciadas en esta región castellana.

Entre 1945-1996 no se aprecia una tendencia clara ni homogénea en el comportamiento de las temperaturas anuales, aunque sí una cierta inclinación al aumento de las temperaturas máximas y al descenso de las temperaturas mínimas y medias. No obstante, si se analizan las series estacionalmente sí que se existe una tendencia al aumento de las anomalías de temperatura durante el invierno y el verano (tanto de anomalías de las máximas como de las medias y mínimas), y al descenso en las estaciones equinocciales.

Pero si este intervalo temporal se analiza en dos subseries (1945-1971 y 1972-1996), los resultados son bien distintos y hasta contrapuestos. En el primer período no hay tendencia significativa, salvo al descenso de las temperaturas mínimas; en cambio, en el segundo período las series muestran una tendencia clara al aumento de las anomalías a un nivel de confianza del 95\%. Esta circunstancia se confirma en los valores estacionales, sobre todo de primavera y verano y secundariamente de invierno y otoño (CUADRO 3).

Entre 1972 y 1996 hay una tendencia creciente de los valores de las anomalías de las temperaturas máximas, mínimas y medias, tanto anuales como estacionales, que se puede promediar en torno a $0.05^{\circ} \mathrm{C} / \mathrm{año}$ (MORALES et al. 
2005). Esto concuerda con los resultados obtenidos en otras regiones españolas y europeas (SCHÖNWISE et al., 1997), aunque cuantitativamente existan diferencias entre los valores de la tendencia (CUADRO 4).

Cuadro 3. Resultados del análisis de tendencia de las series regionales de anomalías de temperatura, en el período 1972-1996

\begin{tabular}{ccccccc}
\hline & & Invierno & Primavera & Verano & Otoño & Año \\
\hline \multirow{3}{*}{$\mathrm{T}^{\text {as }}$ Máximas } & $\mathrm{r}_{\mathrm{s}}$ & 0.4169 & 0.5038 & 0.4015 & 0.0554 & 0.5462 \\
\cline { 2 - 8 } & $\alpha_{\mathrm{s}}$ & 0.038 & 0.010 & 0.047 & 0.793 & 0.005 \\
\cline { 2 - 7 } & tendencia & $\mathbf{C}$ & $\mathbf{C}$ & $\mathbf{C}$ & $\mathbf{N T}$ & $\mathbf{C}$ \\
\hline \multirow{3}{*}{$\mathrm{T}^{\mathrm{a}}$ Mínimas } & $\mathrm{r}_{\mathrm{s}}$ & 0.2738 & 0.6638 & 0.4977 & 0.5162 & 0.6585 \\
\cline { 2 - 7 } & $\alpha_{\mathrm{s}}$ & 0.185 & 0.000 & 0.011 & 0.008 & 0.000 \\
\cline { 2 - 7 } & tendencia & $\mathbf{N T}$ & $\mathbf{C}$ & $\mathbf{C}$ & $\mathbf{C}$ & $\mathbf{C}$ \\
\hline \multirow{3}{*}{$\mathrm{T}^{\mathrm{as}}$ Medias } & $\mathrm{r}_{\mathrm{s}}$ & 0.3862 & 0.6377 & 0.4800 & 0.3092 & 0.6562 \\
\cline { 2 - 7 } & $\alpha_{\mathrm{s}}$ & 0.057 & 0.001 & 0.015 & 0.133 & 0.000 \\
\cline { 2 - 7 } & tendencia & $\mathbf{N T}$ & $\mathbf{C}$ & $\mathbf{C}$ & $\mathbf{N T}$ & $\mathbf{C}$ \\
\hline
\end{tabular}

$\mathrm{r}_{\mathrm{s}}=$ Coeficiente de Spearman; Nivel de confianza $=95 \%, \alpha_{0}=0.05 ; \alpha_{\mathrm{s}}=$ nivel de probabilidad de la hipótesis nula (no existe tendencia). Tendencia: $\mathrm{C}=$ Creciente; $\mathrm{D}=$ Decreciente; NT=no tendencia.

FUENTE: MORALES et al. (2005).

Cuadro 4. Valores de los coeficientes de tendencia anuales para el periodo 19721996

\begin{tabular}{lccccc}
\hline & $\begin{array}{c}\text { Invierno } \\
{ }^{\circ} \text { C/año }\end{array}$ & $\begin{array}{c}\text { Primavera } \\
{ }^{\circ} \text { C/año }\end{array}$ & $\begin{array}{c}\text { Verano } \\
{ }^{\circ} \text { C/año }\end{array}$ & $\begin{array}{c}\text { Otoño } \\
{ }^{\circ} \text { C/año }\end{array}$ & $\begin{array}{c}\text { Año } \\
{ }^{\circ} \text { C/año }\end{array}$ \\
\hline $\mathrm{T}^{\text {as }}$ Máximas & 0.053 & 0.070 & 0.069 & 0.037 & 0.057 \\
\hline $\mathrm{T}^{\text {as }}$ Mínimas & 0.018 & 0.045 & 0.045 & 0.052 & 0.040 \\
\hline $\mathrm{T}^{\mathrm{as}}$ Medias & 0.036 & 0.058 & 0.057 & 0.044 & 0.049 \\
\hline
\end{tabular}

FUENTE: MORALES et al. (2005).

De ellas, han sido las temperaturas máximas de primavera y verano las que han experimentado un mayor aumento con valores de $0.07^{\circ} \mathrm{C} / \mathrm{año}$ mientras que las que han mostrado un aumento menor han sido las mínimas de invierno con tan sólo $0.02^{\circ} \mathrm{C} /$ año (CUADRO 4).

Por otro lado, la aplicación de la metodología seguida respecto al análisis de los valores térmicos extremos, ha proporcionado unos valores umbral, por debajo o por encima de los cuales se pueden considerar temperaturas extremadamente bajas o altas respectivamente en cada una de las submesetas del interior peninsular (CUADRO 5). Así, por ejemplo, para el caso de Castilla y León se define como días calurosos aquellos que superan los $31,3^{\circ} \mathrm{C}$ de máxima y los $17,8^{\circ} \mathrm{C}$ de mínima; y como días muy fríos aquellos cuya máxima esté por debajo de $5,3^{\circ} \mathrm{C}$ y su mínima por debajo de $-3,3^{\circ} \mathrm{C}$.

A partir de estos umbrales se construyen las series de frecuencias estacio- 
nales y anuales de valores extremos de temperaturas máximas y mínimas, y se analizan sus tendencias (FIGURA 6).

Cuadro 5. Valores umbral de temperaturas extremas en el intervalo 1961-2003

\begin{tabular}{lcccc}
\hline \multirow{2}{*}{ Variables } & \multicolumn{2}{c}{ Submeseta Norte } & \multicolumn{2}{c}{ Submeseta Sur } \\
\cline { 2 - 5 } & $\mathrm{P}_{05}\left({ }^{\circ} \mathrm{C}\right)$ & $\mathrm{P}_{95}\left({ }^{\circ} \mathrm{C}\right)$ & $P_{05}\left({ }^{\circ} \mathrm{C}\right)$ & $P_{95}\left({ }^{\circ} \mathrm{C}\right)$ \\
\hline Temperatura máxima & 5,33 & 31,3 & 8,1 & 34,5 \\
\hline Temperatura mínima & $-3,32$ & 17,85 & $-2,0$ & 18,3 \\
\hline
\end{tabular}

FUENTE: ORTEGA et al., 2006 (Submeseta Norte); LABAJO et al., 2006a (Submeseta Sur).

Figura 6. Evolución temporal de la frecuencia anual de los valores más bajos y altos de anomalías de temperatura máxima entre 1961-2003
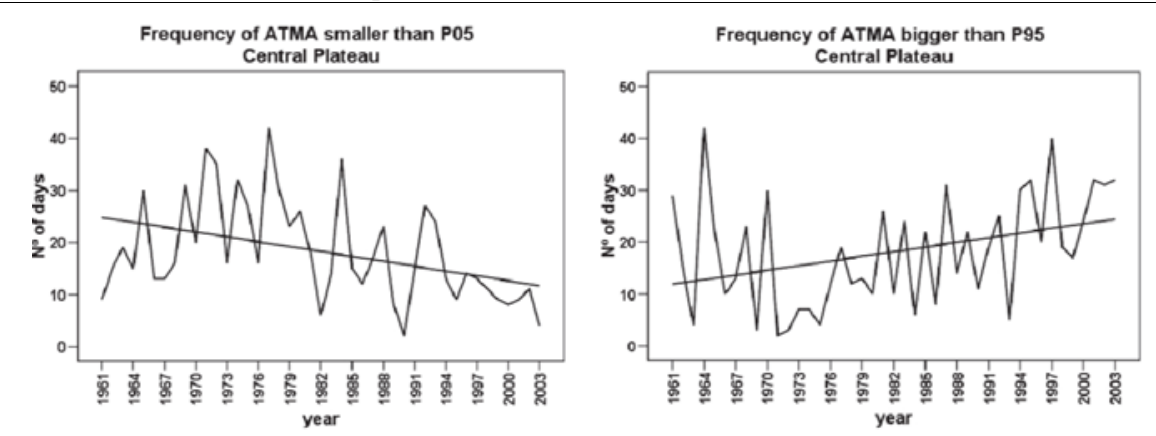

FUENTE: LABAJO et al. (2012)

Se observa que los valores más bajos de temperatura máxima diaria presentan una tendencia anual decreciente mientras que los valores más elevados tienen una frecuencia creciente. Esto viene a decir que el número de días al año con temperaturas máximas más altas en el período analizado se está incrementando, mientras disminuye el número de días con máximas más bajas. Estacionalmente son la primavera y el verano las que más influyen en el comportamiento anual de las frecuencias de los valores extremos de temperatura máxima diaria.

Por su parte, las temperaturas mínimas diarias muestran resultados similares, los valores más bajos tienen una frecuencia anual decreciente y los más altos creciente, sobre todo en el período 1971-2003 (con coeficientes de confianza en torno al 100\%). Y estas mismas tendencias, tanto para las máximas como para las mínimas, se verifican en la submeseta sur.

Pero estos resultados no deben entenderse como algo aislado o exclusivo del interior peninsular ibérico, pues coinciden con lo obtenido en otros trabajos previos (SALINGER et al., 2001; MANTON et al., 2001).Se puede aceptar que la tendencia creciente de la temperatura media anual se debe fundamen- 
talmente a un aumento (tanto en las máximas diarias como en las mínimas diarias) de la frecuencia anual de los casos extremos más altos y a una disminución de la frecuencia anual de los casos extremos más bajos.

Una hipótesis que podría explicar las tendencias observadas es considerar la posibilidad de un incremento en el número de días despejados (o con nieblas), ante el mayor dominio de situaciones dinámicas de carácter anticiclónico, a expensas de una disminución en el número de días nubosos o cubiertos a lo largo del año asociados a situaciones inestables. Por este motivo se ha puesto en relación la evolución de las temperaturas con la que han experimentado los valores de presión atmosférica (LABAJO et al., 2004) en este mismo intervalo temporal, cuyos resultados se exponen más adelante. También cabe pensar que las situaciones anticiclónicas invernales tan características de estas regiones por los tipos de tiempo tan fríos que generan (sobre todo si permiten la aparición de nieblas), no estén produciendo situaciones de frío tan intensas como en otros períodos. Es algo que habrá que investigar.

Los últimos trabajos se han centrado en el análisis de las tendencias de las frecuencias de las olas de frío y de olas de calor que se han desarrollado en el interior peninsular desde 1961 a 2010. Al respecto de estos episodios temporales de condiciones climáticas más extremas, hay que decir que no existe una definición precisa ni universalmente aceptada. Su definición se establece más por la incidencia que tienen sobre el hombre y sus diversas actividades (CHANGNON et al., 2003; ROBINSON, 2001; NOGUEIRA y PAIXÃO, 2008), que no por los valores de las variables meteorológicas, principalmente las temperaturas.

Las definiciones manejadas, líneas atrás justificadas, no se ajustan estrictamente con la percepción habitual que al respecto tiene el ser humano (larga prolongación de días muy fríos o muy calurosos). No se trata de restringir las olas de frío al período invernal y las olas de calor al estival, sino de comprobar la frecuencia y la tendencia que muestra la sucesión de días con temperaturas máximas y mínimas diarias suficientemente bajas (olas de frío) y la sucesión de días con temperaturas máximas y mínimas diarias suficientemente altas (olas de calor) a lo largo de todo el año. Asumiendo que este análisis no incorpora todos los matices climáticos que definen una auténtica ola de frio o de calor, es un primer paso en su definición meteorológica y estadística.

Los resultados referentes a las olas de frío indican que todos los meses del año son susceptibles de sufrir este tipo de procesos (según se ha definido el concepto ola de frío), y que el número total de episodios detectado asciende a 150, de muy diferente duración. Los años con mayor número de ellas han 
sido 1969, 1971 y 1977 y los de menor 1968 y 1989. Las olas más intensas tienen su mayor frecuencia anual en 1971 y 1984, con cuatro episodios cada uno. Las olas de más de cuatro días de duración suponen el 25,3\% del total; las de más de 6 días, el 7,3\%. Estas últimas son las tradicionalmente conceptuadas como auténticas olas de frío, aquellas que se producen cada cinco o siete años, asociadas a la concatenación de varios tipos de tiempo muy fríos (vaguadas de aire ártico (Am), circulaciones retrógradas (aire Pc), gotas frías polares y árticas (Pm, Am), cuñas anticiclónicas muy marcadas y centradas en la Península Ibérica...

El análisis temporal de las series de frecuencias anuales indica que existe una tendencia decreciente a un nivel de confianza del 99\%. Si se considera un modelo lineal $(\operatorname{Fr}(\mathrm{F})=-0,054 *$ año $+4,367)$ en su comportamiento temporal en las cuencas sedimentarias del interior de España se advierte que existe una disminución en la frecuencia de producción de las olas de frío a lo largo del año del orden de 0,54 cada década. Un resultado esperable y en concordancia con las tendencias de las temperaturas máximas y mínimas diarias.

Por su parte, las olas de calor igualmente son susceptibles de acontecer a lo largo de todos los meses del año, si bien no con igual frecuencia ni intensidad. Su número total en ambas mesetas se eleva a 124, de las que 64 han sido más intensas. Los años con mayor número han sido 2005, 2006 y 2009; los que no se han detectado olas 1963, 1967, 1971, 1972, 1975 y 1976. Los meses con mayor número de ellas son mayo y junio, seguidos de abril y agosto. Las olas de más de cuatro días de duración representan el 30,6\% del total, las de más de 6 días el 11,3\%. Estas últimas se corresponden con las olas más excepcionales, aquellas que se producen cada mayor número de años asociadas a penetraciones muy intensas y prolongadas de aire sahariano (Tc) desde el norte de África, que generan mayores impactos sobre la población.

Las líneas de tendencia establecidas a partir de un modelo lineal $\operatorname{Fr}(\mathrm{C})=$ 0,056*año - 1,052) indica que existe una tendencia creciente a un nivel de confianza superior al 99\%. Dicho modelo establece que se ha producido un aumento en la frecuencia de olas de calor en ambas Castillas del orden de 0,6 olas cada década.

Actualmente estos trabajos se van a complementar con la cuantificación de la intensidad de las olas y el análisis de su comportamiento temporal, aplicándolo a escalas espaciales más reducidas y para diferentes épocas del año. 


\section{IV.2. En las precipitaciones}

Una de las variables climáticas que plantea más dificultades al tratar de establecer su comportamiento espacio-temporal es la precipitación. Las razones de estas dificultades son múltiples destacándose entre otras, el grado de exactitud de la medida; la alta variabilidad de su distribución espacial, aún en áreas de características geográficas muy uniformes, consecuencia de sus diferentes orígenes; y su comportamiento temporal a lo largo del año, determinado por la actuación de las perturbaciones del campo bárico en cada zona de la Tierra.

Los análisis de tendencias llevados a cabo en el ámbito de Castilla y León no permiten establecer la existencia de una tendencia clara entre 1945 a 1996, como en el caso de las temperaturas. Pese a todo puede admitirse una cierta tendencia creciente de las anomalías en los primeros años, en el centro del intervalo no existe tendencia mínimamente apreciable, y en los últimos años la tendencia es decreciente y algo más acusada.

Analizando su comportamiento en los dos subperiodos que las temperaturas nos dejaban ver tan claramente (1945-1971 y 1972-1996), los resultados de las precipitaciones no muestran tendencias significativas en ninguno de ellos. Bien es cierto que si se utilizan niveles de significación más pequeños si se aprecia una tendencia decreciente, sobre todo en las últimas décadas. Por ello, se han generado series correspondientes a medias móviles de 30 años (intervalo normalizado por la OMM), y los resultados muestran que las anomalías de precipitación sí que presentan, sobre todo a partir de 1987, una tendencia decreciente de las series anuales, de las de otoño y de las de invierno. Por lo tanto, los valores de las anomalías de precipitación son siempre menores que los de 30 años antes.

Desde luego que los resultados no permiten asegurar, ni cuantificar, la existencia de tendencias en la precipitación zonal, anual y estacional, pero confirman los indicios de la posible disminución de la cantidad de precipitación recogida en las últimas décadas. Estos mismos indicios se confirman si se correlacionan las series de anomalías regionales anual y estacional de precipitaciones y la presión atmosférica mediante técnicas de regresión lineal (CUADRO 6).

Cuadro 6. Coeficiente de correlación (r) y nivel de confianza de las regresiones lineales entre las series regionales de anomalías de precipitación y de presión

\begin{tabular}{lccccc}
\hline & Invierno & Verano & Otoño & Primavera & Año \\
\hline $\mathrm{r}$ & $-0,706$ & $-0,283$ & $-0,758$ & $-0,762$ & $-0,679$ \\
\hline n.c. $(\%)$ & $>99$ & $>95$ & $>99$ & $>99$ & $>99$ \\
\hline r & $>99$ & & &
\end{tabular}

FUENTE: LABAJO Y PIORNO (2001). 
La correlación es inversa, es decir, que el comportamiento de las anomalías de precipitación es contrario al de las anomalías de presión. Por tanto, se ha comprobado que las series de anomalías anuales y estacionales de presión presentan tendencias crecientes en el intervalo 1972-1994, mientras que las series de anomalías anuales y estacionales de precipitación también presentan tendencia, pero decreciente.

La menor correlación que se da en verano es consecuencia del tipo de precipitación que se produce en esta época del año, pues predomina la precipitación de tipo convectivo sobre la de tipo frontal, siendo esta última la responsable de los principales aportes pluviométricos en el área de estudio.

Este comportamiento se justificaría, desde un punto de vista dinámico y ligándolo a la circulación general atmosférica como hacen otros investigadores (RODRIGO et al. 2007), por una posible elevación en latitud de la posición media de las trayectorias de las borrascas asociadas al frente polar.

\section{IV.3. En la presión}

Aunque la presión atmosférica es un elemento climático de primer orden pues determina el comportamiento de otras variables meteorológicas, sus tendencias a lo largo del tiempo han sido poco estudiadas. Generalmente se han realizado estudios de reconstrucción de sus series (BÄRRING et al, 1999), cambios temporales o en relación con otras variables (LABAJO et al., 1998, 2001), si bien más recientemente se han incrementado los estudios regionales para confirmar los resultados de análisis globales, y también se ha abordado el comportamiento de sus valores extremos a nivel del suelo, a partir de series de datos diarios (LABAJO et al. 2008).

El análisis del comportamiento temporal de la presión atmosférica media anual en Castilla y León muestra, al igual que en otros trabajos, la existencia de un cambio nítido a partir del año 1972. Antes de este punto de inflexión y desde 1945 no se advierte ninguna tendencia clara. Sin embargo a partir de este año y hasta 1994 existe una tendencia al aumento, estimándose la variación media anual en 0,06 mmHg/año (LABAJO et al., 1998), un valor pequeño aunque significativo, y que hay que suponer que no pueda aumentar indefinidamente como es lógico. Cabe esperar por tanto, variaciones en esta tendencia en el futuro. Es un tema en curso de actualización.

Los primeros resultados del análisis del comportamiento extremo de esta variable nos llevan a identificar como valores umbral los definidos por los percentiles $\mathrm{P}_{05}$ y $\mathrm{P}_{95} \mathrm{y}$ que aparecen expresados en el CUADRO 7. Es decir, aquellos por debajo o por encima de los cuales se pueden considerar niveles de presión extremadamente bajos o altos respectivamente. 
A partir de estos umbrales, desde la serie regional de anomalías de presiones máximas y mínimas se construyen las series de frecuencias anuales por encima y por debajo de los valores correspondientes (CUADRO 7). Seguidamente se analizan las tendencias de las frecuencias de los valores extremos de presión máxima y mínima. Los resultados obtenidos muestran que la frecuencia anual de valores extremos más bajos de las anomalías de presión máxima (anpmax $<\mathrm{P}_{05}$ ) tienden a disminuir, mientras que los valores extremos más altos (anpmax $>\mathrm{P}_{95}$ ) tienden a aumentar (CUADRO 8).

Cuadro 7. Valores umbrales extremos de anomalías de Presión (intervalo 19741995)

\begin{tabular}{ccc}
\hline Variables & $\mathrm{P}_{05}(\mathrm{hPa})$ & $\mathrm{P}_{95}(\mathrm{hPa})$ \\
\hline Anomalía de Presión máxima & $-11,47$ & 10,12 \\
\hline Anomalía de Presión mínima & $-9,88$ & 8,82 \\
\hline
\end{tabular}

FUENTE: LABAJO et al. (2004).

Por su parte, las tendencias de las anomalías de presión mínima indican que el número anual de casos de anomalías de presión mínima diaria más baja (anpmin $<\mathrm{P}_{05}$ ) disminuye, mientras que el número de casos de anomalías de presión mínima diaria más altas (anpmin $>\mathrm{P}_{95}$ ) aumentan (CUADRO 8).

Cuadro 8. Tendencias de las anomalías de Presión máxima y mínima

\begin{tabular}{ccccccc}
\hline & Anomalías & $r_{s}$ & $u\left(r_{s}\right)$ & $\alpha_{s}$ & $N^{o}$ años & Tendencia \\
\hline \multirow{2}{*}{ Máxima } & anpmax $<\mathrm{P}_{05}$ & $-0,4798$ & 2,1987 & 0,02 & 22 & $\mathrm{D}$ \\
\cline { 2 - 7 } & anpmax $>\mathrm{P}_{95}$ & 0,5375 & 2,4631 & 0,01 & 22 & $\mathrm{C}$ \\
\hline \multirow{2}{*}{ Mínima } & anpmin $<\mathrm{P}_{05}$ & $-0,4110$ & 1,8834 & 0,057 & 22 & $\mathrm{D}$ \\
\cline { 2 - 7 } & anpmin $>\mathrm{P}_{95}$ & 0,6257 & 2,8756 & 0,002 & 22 & $\mathrm{C}$ \\
\hline
\end{tabular}

FUENTE: LABAJO et al. (2004).

Posteriormente se amplió el período de análisis de la presión máxima y mínima al período comprendido entre 1961-2003, y se aumentó el ámbito territorial a las dos submesetas del interior peninsular (LABAJO et al. 2008 y 2009), apreciándose ligeros ascensos en los valores umbral, no así en los resultados de las tendencias. Se sigue manteniendo una tendencia decreciente en la frecuencia anual de los valores más bajos de la presión máxima extrema a nivel del suelo $\left(r_{s}=-0,410\right)$ y una tendencia creciente en el caso de los valores extremos más altos $\left(\mathrm{r}_{\mathrm{s}}=0,259\right)$. Por su parte, los valores más bajos de presión mínima diaria presentan una tendencia decreciente $\left(\mathrm{r}_{\mathrm{s}}=-0,454\right)$ mientras que los más altos la presentan creciente $\left(\mathrm{r}_{\mathrm{s}}=0,437\right)$.

Se advierte en casi todos los observatorios que los años 1963 y 1996 han tenido las frecuencias más elevadas de días con niveles de presión tanto máximas como mínimas más bajos (días extremadamente bajos, umbral $\mathrm{P}_{05}$ ). En cambio, el año 1983 ha sido el que ha mostrado la frecuencia más elevada de 
días con presiones máximas más altas (valores extremadamente altos, umbral $\mathrm{P}_{95}$ ). En cuanto a la presión mínima, si bien existe una mayor variabilidad, también han destacado los años 1990 y 1998. Estacionalmente se comprueba que es el invierno el que determina el comportamiento observado en la presión máxima y mínima anual, pues el resto de períodos estacionales no muestran ningún efecto apreciable al no tener ninguna tendencia en su comportamiento temporal, salvo la primavera en la submeseta norte para la presión mínima. Así pues, son los meses de diciembre, enero y febrero los que revelan una mayor probabilidad de ocurrencia de eventos ciclónicos en el Atlántico.

Figura 7. Evolución temporal de la frecuencia anual de los valores más bajos y altos de anomalías de temperatura máxima entre 1961-2003

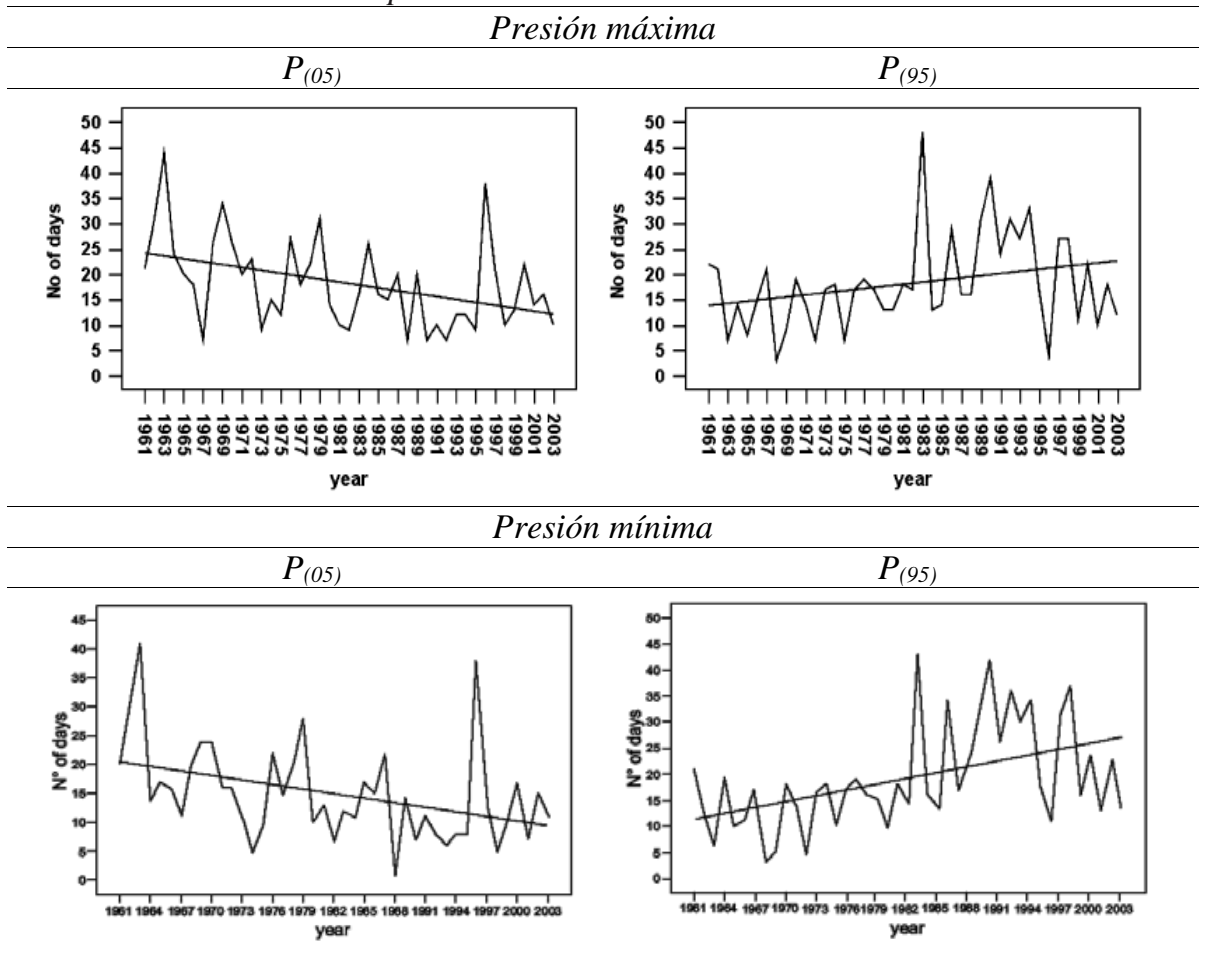

FUENTE: LABAJO et al. 2008 (imagen superior) y LABAJO et al. 2009 (imagen inferior).

El comportamiento de los eventos extremos de presión máxima y mínima en los meses de verano se ve afectado por la existencia de una baja térmica que afecta a la zona de estudio, aunque principalmente en la zona sur, mostrando tendencias decrecientes. Esta baja térmica es un factor determinante 
en ese cambio de signo de la tendencia de los valores más altos de las frecuencias de anomalías de presión máxima y mínima en este territorio.

Se aprecia a lo largo del año desde 1961 a 2003 una tendencia a aumentar el número de días con presiones máximas muy altas, mientras que el número de días con presiones máximas muy bajas tiende a disminuir, circunstancia que también se aprecia para el caso de la presión mínima (FIGURA 7). Todo ello implica un posible aumento de las situaciones anticiclónicas a expensas de una disminución de eventos ciclónicos a lo largo del año. Estos resultados están en acuerdo con los obtenidos en el comportamiento temporal de otras variables, en particular, la temperatura media anual del aire.

Por otra parte, el comportamiento temporal de los valores extremos de presión máxima, en cuanto a la frecuencia de su ocurrencia anual, está mejor definida en la submeseta norte que en la sur. Mientras en Castilla y León la tendencia de la frecuencia anual de los valores de presión más altos y más bajos muestran un nivel de confianza superior al 99\%, en la Submeseta sur este nivel es de más del 95\% para los valores más bajos pero de menos del 90\% para los valores más altos. Aspecto que puede deberse a la forma de penetración de los sistemas ciclónicos en la Península Ibérica, desde el oeste, afectando más a su mitad septentrional que a la meridional. En el caso de la presión mínima los niveles de confianza alcanzados son en todos los casos del 99\%.

\section{CONCLUSIONES}

Las investigaciones climáticas llevadas a cabo por el grupo de Cambio Climático de las universidades de Salamanca y de Valladolid en los últimos 16 años han permitido detectar una serie de cambios en la evolución de las diversas variables meteorológicas analizadas (temperatura, precipitación, presión), al tiempo que ha contribuido a cuantificar la amplitud de los mismos.

La metodología aplicada, tanto en lo referente a la construcción de las series de datos a partir de procesos de homogeneización y regionalización, el trabajo con los valores de anomalía de cada variable y la utilización de los criterios de percentil para el análisis de las variables en sus valores medios y extremos, y de los episodios de olas de frio y de calor, se han mostrado válidos a juzgar por los resultados obtenidos y por la correlación y coincidencia con otros estudios y para diferentes territorios.

Se ha podido concluir que desde el último cuarto del siglo XX se advierte en el sector interior de la Península Ibérica analizado, un aumento de las 
temperaturas medias anuales, fundamentalmente por el comportamiento térmico en la primavera y el verano, pudiéndose ser estimado en $0.05{ }^{\circ} \mathrm{C} / \mathrm{año}$. Análisis posteriores permiten aceptar que esta tendencia creciente se debe fundamentalmente a un incremento (tanto en las máximas como en las mínimas diarias) de la frecuencia anual de los valores extremos más altos y a una disminución de la frecuencia anual de los valores extremos más bajos.

Directamente relacionado con la evolución de las variables térmicas, el comportamiento de la presión atmosférica a nivel del suelo ha permitido comprobar un aumento del número de días al año con presiones máximas cada vez más altas. Esto supondría un posible incremento de situaciones anticiclónicas a expensas de la disminución de dinámicas ciclónicas a lo largo del año.

A diferencia, el análisis de las precipitaciones no muestra unos resultados tan claros pues no se puede asegurar que existan tendencias en la precipitación zonal, anual y estacional, aunque sí se confirman indicios de una posible disminución de la misma en las últimas décadas. Indicios que se confirman al correlacionar las series de anomalías regionales anual y estacional de precipitaciones y la presión atmosférica mediante técnicas de regresión lineal.

Por lo tanto, todo parece indicar que durante las últimas décadas se tiende a un reforzamiento y/o incremento de las situaciones dinámicas anticiclónicas, al menos durante buena parte del año, que son las que están posibilitando la evolución actual de las temperaturas. También cabe pensar que los anticiclones invernales, generadores muchas veces en estas tierras del interior, sobre todo en Castilla y León, de tipos de tiempo muy fríos, sobre todo si permiten la aparición de densas nieblas de irradiación, no estén produciendo situaciones de frío tan intensas como en otros períodos pasados. La evolución que muestran las temperaturas mínimas así lo pone de manifiesto.

Una mayor frecuencia anual de eventos anticiclónicos implica un aumento de la radiación solar que llega a la Tierra a lo largo del año, contribuyendo al aumento detectado en la temperatura del aire en las últimas décadas tanto en estudios globales (JONES et al., 2003; IPCC, 2007) como de escala regional (FORLAND et al. 2001; GALÁN et al., 2001).

La cada vez mayor frecuencia con que se manifiestan los eventos extremos en las diversas variables analizadas, nos ayudan a confirmar la importancia que está teniendo el Cambio Climático en estas regiones del interior de España. 


\section{AGRADECIMIENTOS}

Los resultados obtenidos se han realizado en el marco de varios proyectos de investigación de los Planes Nacionales I+D/I+D+I: Dimensión espacial y temporal del Cambio Climático en España. Análisis de la evolución del comportamiento de los elementos climáticos a partir de datos instrumentales (CLI96-1842-C05-02, 1997-2000). Análisis de la variabilidad espacial y temporal en la ocurrencia de fenómenos térmicos extremos en la meseta norte española. Dimensión espacial y temporal del Cambio Climático en España (REN2000-1769-C06-02; 2000-2001). Tendencias recientes de extremos climáticos en la zona centro de la Península Ibérica. Modelización de la frecuencia de producción de situaciones de riesgo (REN2003-01866; 20032006). Tendencias de la frecuencia temporal de las olas de calor y frío en la zona central de la Península Ibérica. (CGL2009-08565; 2008-2011).

\section{BIBLIOGRAFÍA}

AESAWY, A.M.; HASAMEAN, H.M. (1998): «Annual and seasonal climatic analysis of surface air temperature variations at six southern Mediterranean stations». Theoretical and applied Climatology, $\mathrm{n}^{\circ}$ 61, 55-68.

Alexandersson, H.; Moberg, A. (1997). «Homogenization of Swedish Temperature Data. Part I: Homogeneity Test for Linear Trends». International Journal of Climatology, vol. 17 (1), 25-34.

Allen, R. J. y DeGAETANo, A.T. (2001). «Estimating missing daily temperature extremes using a optimized regression approach». International Journal of Climatology, vol. 21, 1305-1319.

BÄrRING, L.; JÖNSSON, P.; ACHBERGER, C.; EKSTRÖM, M.; AleXANDERSSON, H. (1999). «The Lund instrumental record of meteorological observations: reconstruction of monthly sea-level pressure 1780-1997». International Journal of Climatology, vol. 19 (13), 1427-1443.

BRUnet, M.; Aguilar, E.; SAlAdie, O.; LÓPEZ, D. (1999): «Variaciones y tendencias contemporáneas de la temperatura máxima y mínima y amplitud térmica diaria en el NE de España». La Climatología española en los albores del s. XXI (RASO, J.M.; MARTíN-VIDE, J., eds). Publ. Asociación Española de Climatología (AEC), Serie $A, n^{\circ} 1,113-122$.

BrunetTI, M.; Buffoni, L.; Maugeri, M.; NANNI, T. (2000): «Precipitation intensity trends in northern Italy». International Journal of Climatology, vol. 20 (9), 1017-1031.

CHANGNON, D.; SANDSTORM, M.; SCHAFFER, C. (2003): «Relating changes in agricultural practices to increasing dew points in extreme Chicago heat waves». Climate Research, 24, 243-254.

DEGAETANO, A.T. (1996): «Recent trends in maximum and minimum temperatures threshold excess in the Northeastern United States». Journal of Climate, $n^{\circ} 9$ (7), 1646-1660. 
DeGaetano, A.T.; Allen, R.J. (2002): «Trends in Twentieth-Century Temperature Extremes across the United States». Journal of Climate, $\mathrm{n}^{0}$ 15, 3188-3205.

FORLAND, E.J.; HANSSEN-BAUER, I. (2001): «Changes in temperature and precipitation in the norwegian arctic during the 20th century» en Detecting and Modelling Regional Climate Change (BRUNET et alt. eds.). Springer-Verlag. Berlin, 153161.

GALÁN, E.; CAÑADA, R.; FERNÁNDEZ, F.; CERVERA, B. (2001): «Annual temperature evolution in the southern plateau of Spain from the construction of regional climate time series» en Detecting and Modelling Regional Climate Change (BRUNET et alt. eds.). Springer-Verlag. Berlin, 119-131.

GRIFFITHS G.M.; SALINGER M.J.; LELEU I. (2003): «Trends in extreme daily rainfall across the south Pacific and relationship to the south Pacific convergence zone». I. J. Climatology, $\mathrm{n}^{\circ} 23$ (8), 847-869

InTergovernmental Panel On Climate Change (IPCC) (2001): Third Assessment Report. Climate Change 2001.

InTERgovernmental PANEl On ClimATE CHANGE (IPCC) (2007): Fourth Assessment Report. Climate Change 2007.

JoNES, P.D.; HulME, M. (1996): «Calculating regional climatic time series for temperature and precipitation: methods and illustrations». International Journal of Climatology, vol. 16 (4), 361-377.

JONES, PD.; MOBERG, A. (2003): «Hemispheric and large scale surface air temperature variations: an extensive revision and an update 3 to 2001». Journal of Climate, $\mathrm{n}^{\mathrm{0}} 16,206-223$.

KARL, TH.R.; KNIGHT, R.W.; CHRISTY, J.R. (1994). «Global and Hemispheric Temperature Trends: Uncertainties Related to Inadequate Spatial Sampling». Journal of Climate, $\mathrm{n}^{\circ}$ 7, 1144-1163.g

LABAJO, J.L.; PIORNO, A.; IZQUIERDO, M.J. (1998): «Temporal behavior of the annual mean pressure on the northern Spanish plateau between 1945 and 1994». International Journal of Climatology, vol. 18 (6), 637-647.

LABAJO, J.L., Piorno, A. (1998): «Análisis del comportamiento temporal de las temperaturas en Castilla y León». Clima y ambiente urbano en ciudades ibéricas e iberoamericanas. Ed. Parteluz, 577-592.

LABAJO, J.L., PIORNO, A. (1999): «Comportamiento de variables climáticas en Castilla y León: temperatura mínima media anual» en La Climatología española en los albores del siglo XXI (RASO J.M.; MARTíN-VIDE, J., eds.) I Congreso de la Asociación Española de Climatología (AEC). Serie A, nº 1, 259-266.

LABAJO, J.L., PIORNO, A. (2001): «Regionalization of precipitation in Castilla and León (Spain). Analysis of its temporal behavior» en Detecting and Modeling Regional Climate Change (BRUNET et al. eds.). Springer-Verlag. Berlín, 163-174.

LABAJO, J.L., Piorno, A., Morales, C.G.; OrTEGA, MaT. (2000): "Analysis of the quality of the precipitation data sets in Castilla and Leon. Constructions of a homogeneous basedata». International scientific meeting on detection and modeling of recent climate change and its effects on a regional scale. Climate Change Research Group. Universitat Rovira i Virgili. Tarragona, 12-22.

Labajo, J.L.; Martín, Q.; Piorno, A.; LABAJO, A.L.; Morales, C.G.; OrTeGA, Ma T. (2004): «Primeros resultados del comportamiento de los valores extremos de 
la presión atmosférica al nivel del suelo, en Castilla y León» en El Clima, entre el mar y la montaña (GARCíA, J.C. et al., eds.). Actas del IV Congreso de la Asociación Española de Climatología (AEC). Santander, 313-322

LabAJO, J.L.; LABAJO, A.L.; Piorno, A.; MARTín, Q.; Morales, C.G.; ORTEGA, M. ${ }^{\text {a }}$ T. (2006a): «Análisis del comportamiento reciente de las frecuencias de los valores extremos de temperatura en la zona Madrid-Castilla La Mancha» en Clima, Sociedad y Medio Ambiente (CUADRAT, J.M. et al., eds.). Actas del V Congreso de la Asociación Española de Climatología (AEC). Zaragoza, 157-166.

LABAJO, J.L.; LABAJO, A.L.; MARTíN, Q.; PiORnO, A.; MorAleS, C.G.; ORTEGA, Ma T. (2006b): «Tendencias recientes de los valores extremos de temperatura en la Meseta Sur española». 5ª Asamblea Hispano Portuguesa de Geodesia y Geofísica, CEGG, SPUIAGG e INM. Sevilla.

LABAJO, A.L. (2007): Modelización del comportamiento de la temperatura del aire en superficie usando Redes Neuronales. Tesis Doctoral (Inédita). Universidad de Salamanca. Salamanca.

LABAJO, J.L.; MARTÍN, Q.; LABAJO, A.L.; PiORNO (2007): «Aplicación de un modelo RNA al pronóstico de temperaturas máxima y mínima media mensual en Castilla y León». Actas del XXX Congreso Nacional de Estadística e Investigación Operativa y de las IV Jornadas de Estadística Pública.

Labajo, J.L.; Martín, Q.; LABAJO, A.L.; Piorno, A.; OrTegA, Mª.; Morales, C.G. (2008): «Recent trends in the frequencies of extreme values of daily maximum atmospheric pressure at ground level in the central zone of the Iberian Peninsula», International Journal of Climatology, vol. 28, (9), 1227-1238.

LABAJO, J.L.; LABAJO, A.L.; Piorno, A.; Quintín, M.; ORTEGA, Mª; MoRALES, C.G. (2009): «Analysis of the behavior of the extreme values of minimum daily atmospheric pressure at ground level over the Spanish Central Plateau». Atmósfera, 22 (2), 125-139.

LABAJO, A.L.; LABAJO, J.L. (2011): «Analysis of temporal behavior of climate variables using artificial neural networks: an application to mean monthly maximum temperatures on the Spanish Central Plateau». Atmósfera, 24 (3), 267-285.

LabAJO, J.L.; LABAJO, A.L.; MARTíN, Q.; Morales, C.G.; ORTEGA, Ma T (2012): «Analysis of the maximum daily temperature evolution on the Spanish Central Plateau». Atmósfera, 25 (3), 235-252.

LABAJO, A.L.; MARTÍn, Q.; LABAJO, J.; LABAJO, J.L.; EgIDO, M. (2012a): «Tendencia de las frecuencias de las olas de frío en la meseta central española, entre 1961 y 2010». VIII Congreso Internacional AEC, 431-439

LABAJO, A.L.; EGIDO, M; MARTíN, Q.; LABAJO, J.L.; (2012b): «Evolución temporal de las olas de calor en la meseta central española, entre 1961 y 2010». VIII Congreso Internacional de la AEC. Salamanca, 441-448.

LANA, X.; SERRA, C.; BURGUEÑO, A. (2003): «Trends affecting pluviometric indices at the Fabra Observatory (Barcelona, NE Spain) from 1917 to 1999». International Journal of Climatology, vol. 23 (3), 315-332.

LIEBMANN, B.; JONES, C.; CARVALHO, L.M. (2000): «Interannual variability of daily extreme precipitation events in the state o São Paulo (Brazil)». Journal of Climate, $\mathrm{n}^{\mathrm{o}} 14$ (2), 208-218. 
MAHERAS, P.; KUTIEL, H. (1999): «Spatial and temporal variations in the temperature regime in the Mediterranean and their relations ship with circulation during the last century». International Journal of Climatology, vol. 19 (7), 745-764.

MANTON, M.J.; DELla-MARTA, P.M.; HAYLOCK, M.R. et al. (2001): «Trends in extreme daily rainfall and temperature in Southeast Asia and the South Pacific: 1961-1998». International Journal of Climatology, vol. 21 (3), 269-284.

Morales, C.G.; OrTeGA, Ma T.; LABAJO, J.L.; PiornO, A. (2000): «Analysis of the quality of the series of maximum and minimum temperatures in Castilla and Leon. Construction of a homogeneous base of data». International scientific meeting on detection and modeling of recent climate change and its effects on a regional scale. Climate Change Research Group. Univ. Rovira i Virgili. Tarragona, 23-33.

MORALES, C.G.; ORTEGA, Ma T.; LABAJO, J.L.; PIORNO, A. (2005): «Recent trends and temporal behavior of thermal variables in the region of Castilla-León (Spain)». Atmósfera, n ${ }^{\circ} 18$ (2), 71-90.

NOGUEIRA, P.; PAIXÃO, E. (2008): «Models for mortality associated with heatwaves: update of the Portuguese heat health warning system». International Journal of Climatology, vol. 28 (4), 545-562.

Ortega, Ma T.; Morales, C.G.; LABAJO, J.L.; LABAJO, A.L.; PiornO, A.; Martín, Q. (2006): «Tendencias recientes de las frecuencias de los valores extremos de temperatura en la región de Castilla y León», en Clima, Sociedad y Medio Ambiente (CUADRAT, J.M. et al., eds.). Actas del V Congreso de la Asociación Española de Climatología (AEC). Zaragoza, 251-260.

PINTO, M.; LABAJO, J.L.; PIORNO, A. (2001): «Atmospheric pressure trends between 1945 and 1994 in Castilla and León». Detecting and Modeling Regional Climate Change. Ed. Springer-Verlag, 239-250.

RoBinson, P.J. (2001): «On the definition of a heat wave». Journal of applied Meteorology, $\mathrm{n}^{\circ} 40,762-775$.

RoDRIGO, F.S.; TRIGO, R.M. (2007): «Trends in daily rainfall in the Iberian Peninsula from 1951 to 2002». International Journal of Climatology, vol. 27 (4), 513529

SAliNGER, M.J.; GRIFFITHS, G.M. (2001): «Trends in New Zealand daily temperature and rainfall extremes». International Journal of Climatology, vol. 21 (12), 1437-1452.

SCHÖNWISE, C.D.; RAPP, J. (1997): Climate trends atlas of Europe. Kluwer academic publishers. Dordrecht.

VINNIKOV, K.YA.; GROISMAN, P.YA.; LUGINA, K.M. (1990): «The empirical data on modern global climate changes (temperature and precipitation)». Journal of Climate, 3, 662- 677. 